\title{
WHO recommendations for the prevention and treatment of postpartum haemorrhage
}
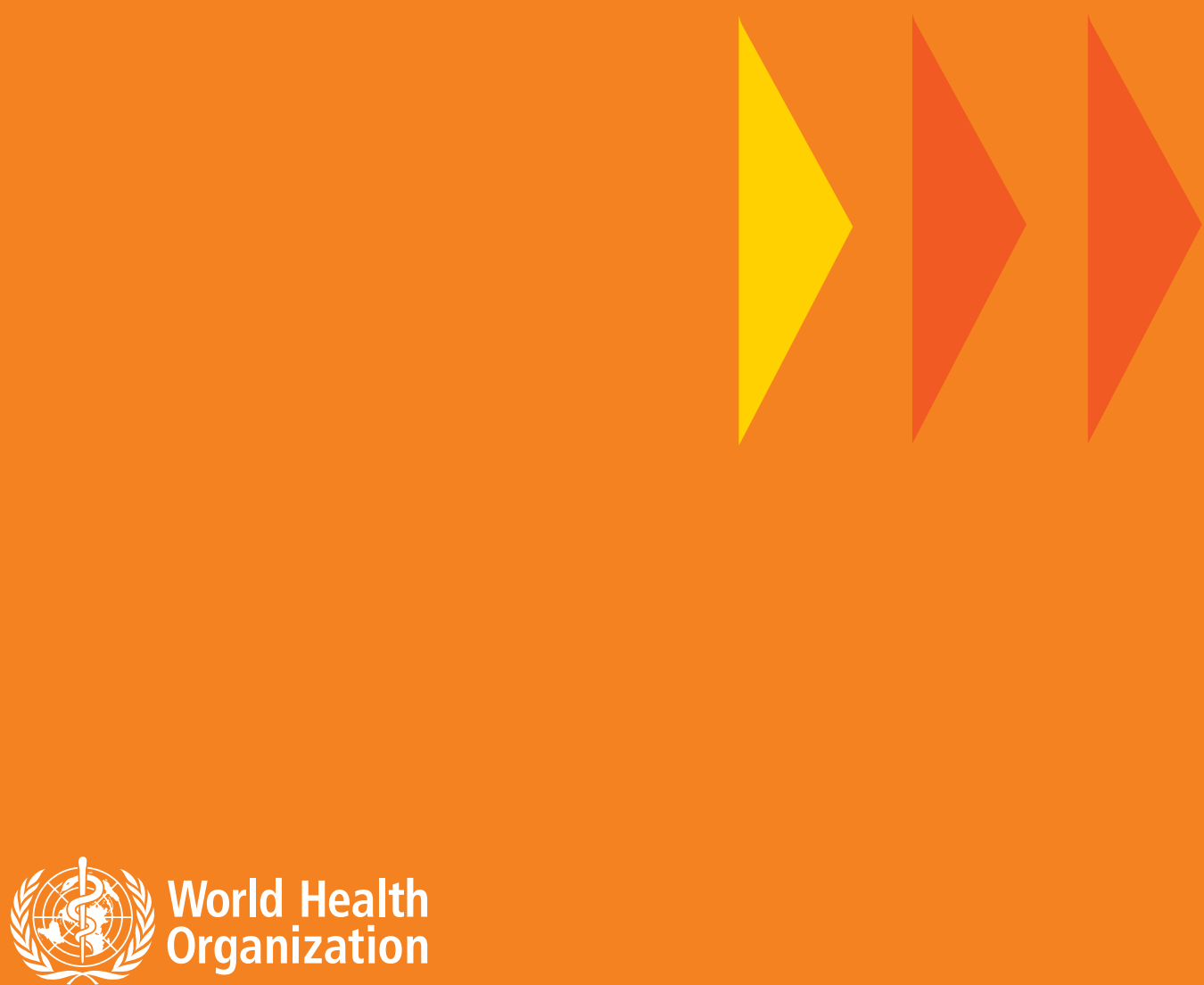



\section{WHO recommendations for the prevention and treatment of postpartum haemorrhage}


WHO Library Cataloguing-in-Publication Data

WHO recommendations for the prevention and treatment of postpartum haemorrhage.

1.Postpartum hemorrhage - prevention and control. 2.Postpartum hemorrhage - therapy. 3.Obstetric labor complications. 4.Guideline. I. World Health Organization.

ISBN 9789241548502

(NLM classification: WQ 330)

(c) World Health Organization 2012

All rights reserved. Publications of the World Health Organization are available on the WHO web site (www.who.int) or can be purchased from WHO Press, World Health Organization, 20 Avenue Appia, 1211 Geneva 27, Switzerland (tel.: +41 22791 3264; fax: +41 22791 4857; e-mail: bookorders@who.int). Requests for permission to reproduce or translate WHO publications - whether for sale or for noncommercial distribution - should be addressed to WHO Press through the WHO web site (http: / www.who.int/ about/licensing/copyright_form/en/index.html).

The designations employed and the presentation of the material in this publication do not imply the expression of any opinion whatsoever on the part of the World Health Organization concerning the legal status of any country, territory, city or area or of its authorities, or concerning the delimitation of its frontiers or boundaries. Dotted lines on maps represent approximate border lines for which there may not yet be full agreement.

The mention of specific companies or of certain manufacturers' products does not imply that they are endorsed or recommended by the World Health Organization in preference to others of a similar nature that are not mentioned. Errors and omissions excepted, the names of proprietary products are distinguished by initial capital letters.

All reasonable precautions have been taken by the World Health Organization to verify the information contained in this publication. However, the published material is being distributed without warranty of any kind, either expressed or implied. The responsibility for the interpretation and use of the material lies with the reader. In no event shall the World Health Organization be liable for damages arising from its use.

Printed in Italy 


\section{Contents}

\begin{tabular}{ll} 
Acknowledgements & 1 \\
\hline
\end{tabular}

\begin{tabular}{ll}
\hline Abbreviations & 2 \\
\hline
\end{tabular}

Executive summary 3

Box A: Recommendations for the prevention of PPH 5

Box B: Recommendations for the treatment of PPH 6

Box C: Recommendations on organization of care $\quad 7$

1. Background 8

\begin{tabular}{lr}
\hline 2. Methods 9 \\
\hline 3. Results
\end{tabular}

3. Results 12

Box 1: Recommendations for the prevention of PPH - uterotonics $\quad 15$

Box 2: Recommendations for the prevention of PPH - cord management and uterine massage

Table 1: Recommendation status of the individual components of the active management of the third stage of labour, based on who delivers the intervention 18

Box 3: Recommendations for the prevention of PPH in caesarean sections 18

Box 4: Recommendations for the treatment of PPH - uterotonics 19

Box 5: Recommendations for the treatment of PPH - fluid resuscitation and tranexamic acid

Box 6: Recommendations for the treatment of PPH - manoeuvres and other procedures 20

Box 7: Recommendations for the treatment of retained placenta 21

Box 8: Health Systems and Organization of Care recommendations 22

Box 9: Statements related to topics for which there is insufficient evidence to issue a recommendation 23

\begin{tabular}{ll}
\hline 4. Research implications & 24 \\
\hline 5. Dissemination and
\end{tabular}

5. Dissemination and implementation of the guideline $\quad 25$

\begin{tabular}{ll}
\hline 6. Applicability issues & 26 \\
\hline 7. ppdating the
\end{tabular}

\begin{tabular}{lr}
\hline 7. Updating the guideline & 27
\end{tabular}

\begin{tabular}{ll}
\hline References & 27 \\
\hline
\end{tabular}

Annex 1. External experts, WHO staff involved in the preparation of the guideline, and summary of declarations of interest 29

Annex 2. Critical outcomes for decision making 33

Annex 3: Summary of the considerations related to the strength of the recommendations (Balance Worksheets)

Box 1. Summary of considerations related to the strength of the recommendations (Recommendations 1-5) 
Box 2. Summary of considerations related to the strength of the recommendations (Recommendations 6-10)

Box 3. Summary of considerations related to the strength of the recommendations (Recommendations 11-15)

Box 4. Summary of considerations related to the strength of the recommendations (Recommendations 16-20)

Box 5. Summary of considerations related to the strength of the recommendations (Recommendations 21-25)

Box 6. Summary of considerations related to the strength of the recommendations (Recommendations 26-30)

Box 7. Summary of considerations related to the strength of the recommendations (Recommendations 31-32)

Box 8. Template for the summary of considerations related to the strength of the recommendations with explanations for completing the template

The standardized criteria used in grading the evidence, the narrative summaries of evidence and GRADE tables are not included in this document. This material has been published in a separate document entitled "WHO recommendations for postpartum haemorrhage: evidence base" and can be accessed online at: www.who.int/ reproductivehealth/publications/maternal_perinatal_health/9789241548502/en/ 


\section{Acknowledgements}

Work on this guideline was initiated by A. Metin Gülmezoglu and João Paulo Souza, of the WHO Department of Reproductive Health and Research, and by Matthews Mathai, of the WHO Department of Maternal, Newborn, Child and Adolescent Health. João Paulo Souza coordinated the development of the present guideline and drafted this document. Edgardo Abalos and Virginia Diaz, of the Centro Rosarino de Estudios Perinatales (CREP), Rosario, Argentina, reviewed the scientific evidence related to the prevention and treatment of Postpartum Haemorrhage (PPH) and produced the GRADE tables used in this guideline. Natasha Hezelgrave, of the Academic Women's Health Centre, King's College London (KCL), United Kingdom (UK), drafted the narrative summaries of evidence. The GRADE tables were double-checked by Kanokwaroon Watananirun (Fon) of the University of Bangkok, Thailand. A. Metin Gülmezoglu, Matthews Mathai and Edgardo Abalos commented on the draft document before it was reviewed by Natasha Hezelgrave and participants at the WHO Technical Consultation on the Prevention and Treatment of PPH (see Annex 1).

Thanks to Zahida Qureshi, of the University of Nairobi, Kenya, for serving as the Chairperson of the Technical Consultation. We acknowledge gratefully the valuable feedback given by a large number of international stakeholders during the online consultation which took place as part of this process.

WHO is grateful for the continued support of the United States Agency for International Development (USAID) in this area of work. Special thanks are also due to Gynuity Health Projects for providing additional financial support for this guideline work. WHO also wishes to thank the authors of the systematic reviews used in this guideline for their assistance and collaboration in updating them. WHO is also grateful to the Cochrane Pregnancy and Childbirth Group, especially the staff at their Liverpool office in the United Kingdom, for their support in updating the Cochrane reviews. 


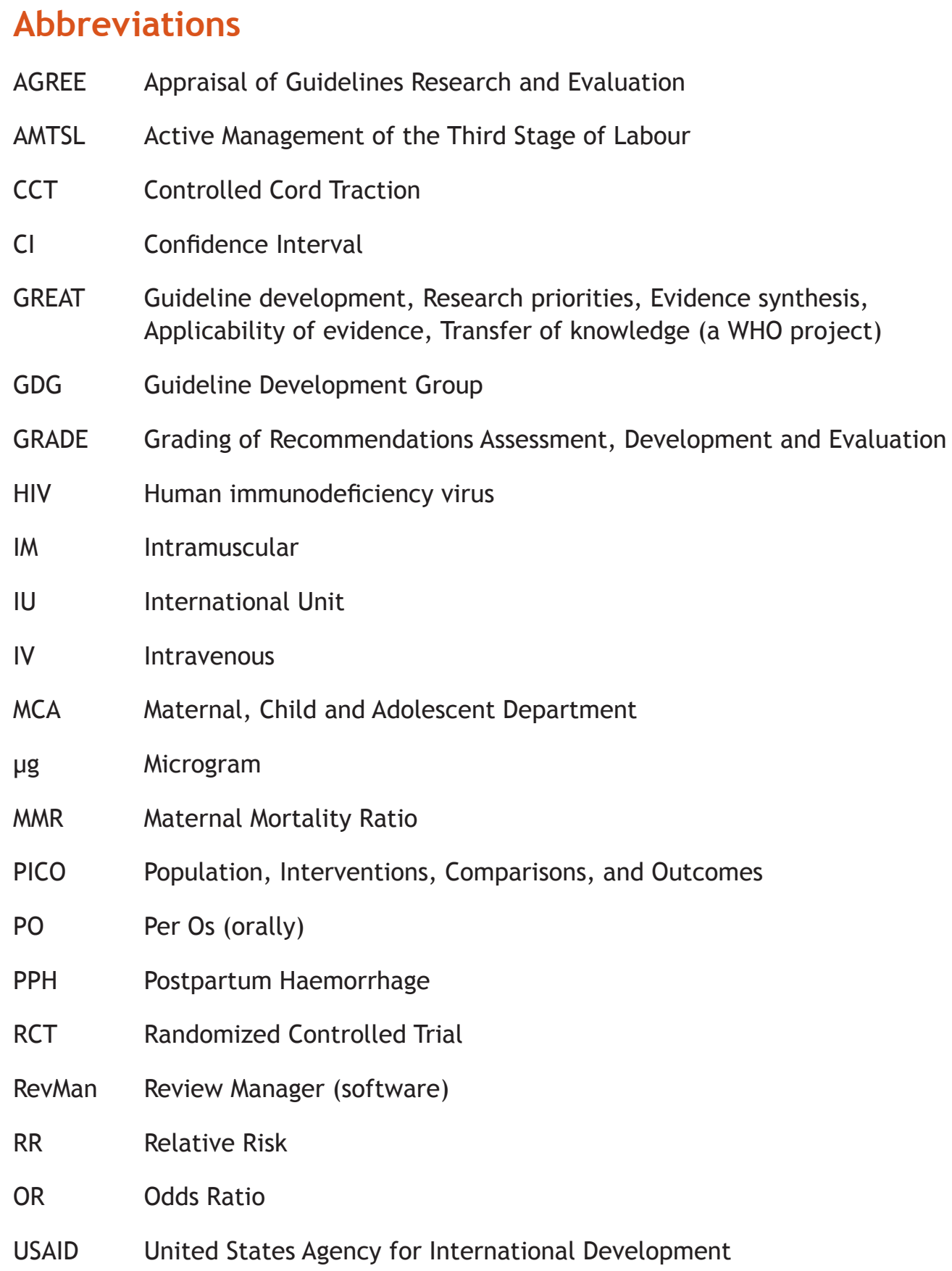




\section{Executive summary}

\section{Introduction}

Postpartum Haemorrhage (PPH) is commonly defined as a blood loss of $500 \mathrm{ml}$ or more within 24 hours after birth. PPH is the leading cause of maternal mortality in low-income countries and the primary cause of nearly one quarter of all maternal deaths globally. Most deaths resulting from PPH occur during the first 24 hours after birth: the majority of these could be avoided through the use of prophylactic uterotonics during the third stage of labour and by timely and appropriate management.

Improving health care for women during childbirth in order to prevent and treat PPH is an essential step towards the achievement of the Millennium Development Goals. The primary objective of this guideline therefore is to provide a foundation for the strategic policy and programme development needed to ensure the sustainable implementation of effective interventions for reducing the global burden of $\mathrm{PPH}$.

\section{Guideline development methods}

The procedures used in the development of this guideline are outlined in the "WHO handbook for guideline development"1. Briefly, these procedures are: (i) the identification of questions related to clinical practice and health policy for which answers are needed, (ii) the retrieval of up-to-date research-based evidence, (iii) the assessment and synthesis of evidence, (iv) the formulation of recommendations using input from a wide range of stakeholders, and (v) the formulation of plans for the dissemination, implementation, impact evaluation and updating of the guideline.

The scientific evidence for the recommendations was synthesized using the Grading of Recommendations Assessment, Development and Evaluation (GRADE) methodology. For each of the previous WHO recommendations on PPH (2007 and 2009) and for all the newly-added questions, evidence profiles were prepared based on 22 up-to-date systematic reviews. The revised and new recommendations were developed and adopted by an international group of experts who participated in the WHO Technical Consultation on the Prevention and Treatment of PPH, held in Montreux, Switzerland, 6-8 March 2012.

The WHO Technical Consultation adopted 32 recommendations and these are shown in Boxes A, B and C. For each recommendation, the quality of the supporting evidence is graded as 'very low', 'low', 'moderate' or 'high'. The contributing stakeholders qualified the strength of these recommendations by taking the quality of the evidence and other factors into account (including the values and preferences of stakeholders, the magnitude of effect, the balance of benefits versus disadvantages, resource usage, and the feasibility of each recommendation). To ensure that each recommendation is correctly understood and used in practice, additional remarks have also been included, and these are noted in the full document below the recommendations. Readers should refer to these remarks in the full version of the guideline if they are in any doubt about the meaning of each recommendation.

1 WHO handbook for guideline development. Geneva, World Health Organization, 2012. 


\section{Recommendations for PPH prevention}

The intrinsic contribution of each component of the 'active management of the third stage of labour' was examined in light of new available evidence, and relevant recommendations were made. All women giving birth should be offered uterotonics during the third stage of labour for the prevention of PPH; oxytocin (IM/IV, 10 IU) is recommended as the uterotonic drug of choice. Other injectable uterotonics and misoprostol are recommended as alternatives for the prevention of PPH in settings where oxytocin is unavailable. The importance of controlled cord traction (CCT) was revisited because of new evidence. This intervention is now regarded as optional in settings where skilled birth attendants are available, and is contraindicated in settings where skilled attendants do not assist with births. Early cord clamping is generally contraindicated. Continuous uterine massage is not recommended as an intervention to prevent PPH in women who have received prophylactic oxytocin, as it may cause maternal discomfort, require a dedicated health professional, and may not lead to a reduction of blood loss. However, surveillance of uterine tonus through abdominal palpation is recommended in all women for early identification of postpartum uterine atony. In summary, the Guideline Development Group (GDG) considered the use of uterotonics as the main intervention within the active management of third stage of labour package. In this context, the use of misoprostol for the prevention of PPH by community health care workers and lay health workers is supported in settings where skilled birth attendants are not present.

The GDG also issued recommendations for reducing blood loss during the third stage of labour in caesarean sections. Oxytocin is the recommended uterotonic drug for the prevention of PPH in caesarean sections. Cord traction is recommended in preference to manual removal when assisting placental delivery in caesarean sections.

\section{Recommendations for PPH treatment}

The use of uterotonics (oxytocin alone as the first choice) plays a central role in the treatment of PPH. Uterine massage is recommended for the treatment of PPH as soon as it is diagnosed, and initial fluid resuscitation with isotonic crystalloids is recommended. The use of tranexamic acid is advised in cases of refractory atonic bleeding or persistent trauma-related bleeding. The use of intrauterine balloon tamponade is recommended for refractory bleeding or if uterotonics are unavailable. Bimanual uterine compression, external aortic compression, and the use of non-pneumatic anti-shock garments are recommended as temporizing measures until substantive care is available. If there is persistent bleeding and the relevant resources are available, uterine artery embolization should be considered. If bleeding persists, despite treatment with uterotonic drugs and other conservative interventions, surgical intervention should be used without further delay.

If the third stage of labour lasts more than 30 minutes, CCT and IV/IM oxytocin (10 IU) should be used to manage the retained placenta. If the placenta is retained and bleeding occurs, the manual removal of the placenta should be expedited. Whenever the manual removal of the placenta is undertaken, a single dose of prophylactic antibiotics is recommended.

The GDG also issued recommendations related to the organization of PPH care. Health facilities delivering maternity services should adopt formal protocols for the prevention and treatment of PPH and for patient referral. The use of PPH treatment 
simulations for pre-service and in-service training programmes was recommended. Finally, the GDG recommended that the use of uterotonics for the prevention of PPH should be monitored and a specific indicator was suggested.

Box A: Recommendations for the prevention of PPH

1. The use of uterotonics for the prevention of PPH during the third stage of labour is recommended for all births. (Strong recommendation, moderate-quality evidence)

2. Oxytocin (10 IU, IV/IM) is the recommended uterotonic drug for the prevention of PPH. (Strong recommendation, moderate-quality evidence)

3. In settings where oxytocin is unavailable, the use of other injectable uterotonics (if appropriate ergometrine/methylergometrine or the fixed drug combination of oxytocin and ergometrine) or oral misoprostol $(600 \mu \mathrm{g})$ is recommended. (Strong recommendation, moderatequality evidence)

4. In settings where skilled birth attendants are not present and oxytocin is unavailable, the administration of misoprostol ( $600 \mu \mathrm{g}$ PO) by community health care workers and lay health workers is recommended for the prevention of PPH. (Strong recommendation, moderatequality evidence)

5. In settings where skilled birth attendants are available, CCT is recommended for vaginal births if the care provider and the parturient woman regard a small reduction in blood loss and a small reduction in the duration of the third stage of labour as important (Weak recommendation, high-quality evidence)

6. In settings where skilled birth attendants are unavailable, CCT is not recommended. (Strong recommendation, moderate-quality evidence)

7. Late cord clamping (performed after 1 to 3 minutes after birth) is recommended for all births while initiating simultaneous essential newborn care. (Strong recommendation, moderatequality evidence)

8. Early cord clamping ( $<1$ minute after birth) is not recommended unless the neonate is asphyxiated and needs to be moved immediately for resuscitation. (Strong recommendation, moderate-quality evidence)

9. Sustained uterine massage is not recommended as an intervention to prevent PPH in women who have received prophylactic oxytocin. (Weak recommendation, low-quality evidence)

10. Postpartum abdominal uterine tonus assessment for early identification of uterine atony is recommended for all women. (Strong recommendation, very-low-quality evidence)

11. Oxytocin (IV or IM) is the recommended uterotonic drug for the prevention of PPH in caesarean section. (Strong recommendation, moderate-quality evidence)

12. Controlled cord traction is the recommended method for removal of the placenta in caesarean section. (Strong recommendation, moderate-quality evidence) 
Box B: Recommendations for the treatment of PPH

13. Intravenous oxytocin alone is the recommended uterotonic drug for the treatment of PPH. (Strong recommendation, moderate-quality evidence)

14. If intravenous oxytocin is unavailable, or if the bleeding does not respond to oxytocin, the use of intravenous ergometrine, oxytocin-ergometrine fixed dose, or a prostaglandin drug (including sublingual misoprostol, $800 \mu \mathrm{g}$ ) is recommended. (Strong recommendation, low-quality evidence)

15. The use of isotonic crystalloids is recommended in preference to the use of colloids for the initial intravenous fluid resuscitation of women with PPH. (Strong recommendation, low-quality evidence)

16. The use of tranexamic acid is recommended for the treatment of PPH if oxytocin and other uterotonics fail to stop bleeding or if it is thought that the bleeding may be partly due to trauma. (Weak recommendation, moderate-quality evidence)

17. Uterine massage is recommended for the treatment of PPH. (Strong recommendation, verylow-quality evidence)

18. If women do not respond to treatment using uterotonics, or if uterotonics are unavailable, the use of intrauterine balloon tamponade is recommended for the treatment of PPH due to uterine atony. (Weak recommendation, very-low-quality evidence)

19. If other measures have failed and if the necessary resources are available, the use of uterine artery embolization is recommended as a treatment for PPH due to uterine atony. (Weak recommendation, very-low-quality evidence)

20. If bleeding does not stop in spite of treatment using uterotonics and other available conservative interventions (e.g. uterine massage, balloon tamponade), the use of surgical interventions is recommended. (Strong recommendation, very-low-quality evidence)

21. The use of bimanual uterine compression is recommended as a temporizing measure until appropriate care is available for the treatment of PPH due to uterine atony after vaginal delivery. (Weak recommendation, very-low-quality evidence)

22. The use of external aortic compression for the treatment of PPH due to uterine atony after vaginal birth is recommended as a temporizing measure until appropriate care is available. (Weak recommendation, very-low-quality evidence)

23. The use of non-pneumatic anti-shock garments is recommended as a temporizing measure until appropriate care is available. (Weak recommendation, low-quality evidence)

24. The use of uterine packing is not recommended for the treatment of PPH due to uterine atony after vaginal birth. (Weak recommendation, very-low-quality evidence)

25. If the placenta is not expelled spontaneously, the use of IV/IM oxytocin (10 IU) in combination with controlled cord traction is recommended. (Weak recommendation, very-low-quality evidence)

26. The use of ergometrine for the management of retained placenta is not recommended as this may cause tetanic uterine contractions which may delay the expulsion of the placenta. (Weak recommendation, very-low-quality evidence)

27. The use of prostaglandin E2 alpha (dinoprostone or sulprostone) for the management of retained placenta is not recommended. (Weak recommendation, very-low-quality evidence)

28. A single dose of antibiotics (ampicillin or first-generation cephalosporin) is recommended if manual removal of the placenta is practised. (Weak recommendation, very-low-quality evidence) 
Box C: Organization of care

29. The use of formal protocols by health facilities for the prevention and treatment of PPH is recommended. (Weak recommendation, moderate-quality evidence)

30. The use of formal protocols for referral of women to a higher level of care is recommended for health facilities. (Weak recommendation, very-low-quality evidence)

31. The use of simulations of PPH treatment is recommended for pre-service and in-service training programmes. (Weak recommendation, very-low-quality evidence)

32. Monitoring the use of uterotonics after birth for the prevention of PPH is recommended as a process indicator for programmatic evaluation. (Weak recommendation, very-low-quality evidence) 


\section{Background}

Postpartum Haemorrhage (PPH) is commonly defined as a blood loss of $500 \mathrm{ml}$ or more within 24 hours after birth, while severe PPH is defined as a blood loss of $1000 \mathrm{ml}$ or more within the same timeframe. PPH affects approximately $2 \%$ of all women who give birth: it is associated not only with nearly one quarter of all maternal deaths globally but is also the leading cause of maternal mortality in most low-income countries. PPH is a significant contributor to severe maternal morbidity and long-term disability as well as to a number of other severe maternal conditions generally associated with more substantial blood loss, including shock and organ dysfunction. (1-3)

Uterine atony is the most common cause of PPH, but genital tract trauma (i.e. vaginal or cervical lacerations), uterine rupture, retained placental tissue, or maternal coagulation disorders may also result in PPH. Although the majority of women who experience PPH complications have no identifiable clinical or historical risk factors, grand multiparity and multiple gestation are associated with an increased risk of bleeding after birth. PPH may be aggravated by pre-existing anaemia and, in such instances, the loss of a smaller volume of blood may still result in adverse clinical sequelae. (4)

During the second half of the 20th century, a package of interventions performed during the third stage of labour became the cornerstone for the prevention of $\mathrm{PPH}$. This approach became known as the "active management of the third stage of labour" and consisted initially of the following components: the administration of a prophylactic uterotonic after the delivery of a baby, early cord clamping and cutting, and the controlled traction of the umbilical cord. Uterine massage is also frequently included as part of the active management of the third stage of labour. In contrast to active management, expectant management involves instead waiting for signs of placenta separation and allows for the placenta to be delivered spontaneously, or aided by nipple stimulation or gravity. Compared with expectant management, the active management of the third stage of labour is associated with a substantial reduction in the occurrence of PPH. (5)

It is generally assumed that by preventing and treating PPH, most PPH-associated deaths could be avoided. The prevention and treatment of PPH are therefore vital steps towards improving the health care of women during childbirth and the achievement of the Millennium Development Goals. To reach these objectives, health workers in developing countries should be given access to appropriate medications and be trained in procedures relevant to the management of PPH. Countries also need evidence-based guidance to inform their health policies and improve their health outcomes.

Given the availability of new scientific evidence related to the prevention and treatment of PPH, the aim of this document is to revise previous WHO recommendations for the prevention and treatment of PPH and to add new recommendations. The primary goal of this guideline is to provide a foundation for the implementation of strategic policy and programme developments for interventions shown to have been effective in reducing the burden of PPH. Health professionals responsible for developing national and local protocols and health policies constitute the main target audience of this document. Obstetricians, midwives, general medical practitioners, health care managers and public health policy-makers, particularly in under-re- 
sourced settings are also targeted. The guidance provided is evidence-informed and covers topics related to the management of PPH that were selected and prioritized by an international, multidisciplinary group of health care workers, consumers and other stakeholders. This document establishes general principles of PPH care and it is intended to inform the development of protocols and health policies related to $\mathrm{PPH}$. This document is not intended to provide a comprehensive practical guide for the prevention and treatment of $\mathrm{PPH}$.

\section{Methods}

This guideline is an update of the "WHO recommendations for the prevention of $P P H$ " published in 2007 and the "WHO guidelines for the management of PPH and retained placenta" published in $2009(6,7)$. This document represents WHO's normative support for using evidence-informed policies and practices in all countries. The guideline forms part of a WHO knowledge-to-action project entitled GREAT (Guideline development, Research priorities, Evidence synthesis, Applicability of evidence, Transfer of knowledge) (8) and was developed using standardized operating procedures in accordance with the process described in the "WHO handbook for guideline development" (9). In summary, the process included: (i) the identification of critical questions and critical outcomes, (ii) the retrieval of the evidence, (iii) the assessment and synthesis of evidence, (iv) the formulation of recommendations, and (v) planning for the dissemination, implementation, impact evaluation and updating of the guideline.

Two technical groups have worked in the development of this guideline. A small operative group composed of staff from the WHO's Department of Reproductive Health and Research, and Department of Maternal, Newborn, Child and Adolescent Health (MCA), as well as two external experts (see Annex 1 - The guideline steering group) and a larger group with international stakeholders including midwives, obstetricians, neonatologists, researchers, experts in research synthesis, experts in health care programmes, and consumer representatives (the Guideline Development Group - GDG). The guideline steering group was formed in the very beginning of the project and reviewed the previous WHO guidelines on prevention and treatment of PPH $(6,7)$. This group prepared a list of potential additional questions related to the prevention and treatment of PPH. Next, the GDG reviewed and prioritized the draft questions. The guideline steering group then produced a list of all the questions to be addressed. This included both questions from the earlier versions of the guideline as well as new ones. The guideline steering group also adopted the outcomes used in the 2007 and 2009 guideline documents. These outcomes, as before, were rated on a scale from 1 to 9 . A question or outcome was defined as 'critical' if it was given an average score of 7 or more. Questions and outcomes with a score of between 4 and 6 were considered 'important but not critical', while those with a score lower than 4 were not considered to be important for the purposes of the guideline (Annex 2). 
Cochrane systematic reviews of randomized controlled trials (RCTs) were the primary source of evidence for the recommendations ${ }^{2}$. Using the assembled list of questions and outcomes, the guideline steering group identified Cochrane systematic reviews that were either relevant or potentially relevant and then evaluated whether any needed updating. A review was considered to be outdated if the last specified date for new trial searches was two years ago or more, or if there were relevant studies still awaiting assessment, as identified by the standard search procedures of the Cochrane Pregnancy and Childbirth Group. Updates were performed using specific standard search strategies. The corresponding authors of the outdated reviews were invited to update them within a specified time period. In instances in which the corresponding authors were unable to do so, the updates were undertaken by members of the guideline steering group. The search strategies employed to identify the trials and the specific criteria for trial inclusion and exclusion are described in the individual systematic reviews. A systematic review of literature that included non-randomized trials was carried out by the guideline steering group members when randomized-trial data related to specific questions were scarce.

The following procedures were used to extract the evidence for this guideline from each of these systematic reviews: first, the most recent version of the Review Manager (RevMan) file was retrieved from the Cochrane Pregnancy and Childbirth Cochrane Group and customized to reflect the key comparisons and outcomes (those that were not relevant to the guideline were excluded). Then the RevMan file was exported to the GRADE profiler software (Grading of Recommendations Assessment, Development and Evaluation) and GRADE criteria were used to critically appraise the retrieved scientific evidence. Finally, evidence profiles (in the form of GRADE tables) were prepared for each comparison. An online content management system developed for the GREAT project, namely the Guideline Production System, was used to handle and share electronic files.

The evidence presented in the GRADE tables was derived from a larger body of data extracted primarily from Cochrane reviews which, in many cases, contained multiple comparisons (Evidence Base (EB) Tables 1 to 70). Each GRADE table relates to one specific question or comparison, but some GRADE tables do not contain data for all critical outcomes. This is because data for those outcomes were not available in the Cochrane reviews. The raw data which constitute the basis of the GRADE tables are not included in this document, but readers interested in how these GRADE tables were constructed may request access to this information. The guideline steering group used the information presented in the GRADE tables to check if any existing recommendations (included in the 2007 or 2009 documents) needed to be revised, and to draft recommendations that related to the new questions. Each recommendation was allocated to a thematic module which included the narrative summaries of evidence and the relevant GRADE tables. The standardized criteria used in grading the evidence and the thematic modules (including the GRADE tables) are not included in this document. They have been published separately online in a document entitled "WHO recommendations for preventing and treating PPH: evidence base" (www.who.int/reproductivehealth/publications/maternal_perinatal_health/9789241548502/en).

\footnotetext{
2 As part of the Cochrane pre-publication editorial process, reviews are commented on by three peers (one editor and two referees external to the editorial team) and the Group's Statistical Adviser (see http:/ / www.cochrane.org/cochrane-reviews). "The Cochrane Handbook for Systematic Reviews of Interventions" describes in detail the process of preparing and maintaining Cochrane systematic reviews on the effects of health care interventions.
} 
A preliminary online consultation was held to review the draft recommendations. The draft recommendations and supporting evidence were made available to a large number of international stakeholders who were then asked to respond to an online survey. In addition, the preliminary online consultation identified other previous recommendations that needed to be discussed at the WHO Technical Consultation on the Prevention and Treatment of PPH held in Montreux, Switzerland, 6-8 March 2012. A subset of the international group of experts (who had participated in the online consultations) and other additional experts were invited to attend the Technical Consultation (see Annex 1 for a full list of participants). The draft recommendations, the narrative summaries of evidence, the GRADE tables for the new and previous recommendations, and other related documents were provided in advance to participants. Balance worksheets were used during the Technical Consultation to summarize the values, preferences and judgements made about the strength of the new and revised recommendations.

\section{Declaration of interest by participants in the WHO Technical Con- sultation}

According to WHO regulations, all experts must declare their relevant interests prior to participation in WHO meetings. All GDG members and participants were therefore required to complete a Declaration of Interest Form before the meeting. These were reviewed by the guideline steering group before the group composition and invitations were finalized. The external advisers also verbally declared potential conflicts of interest at the beginning of the meeting. The procedures for the management of conflicts of interests were undertaken in accordance with the "WHO guidelines for declaration of interests (WHO experts)". In summary, all members of the GDG declared that they had no commercial or financial interests that were directly or indirectly related to the topic of the meeting/guideline. Seven members of the GDG were involved in academic work related to the topic of the guideline, but this involvement was not considered to be a conflict of interest and the full participation of all the selected experts was deemed appropriate. A table summarizing the declarations of interest made by members of the GDG is included in Annex 1.

\section{Decision-making during the Technical Consultation}

At the beginning of the Technical Consultation, the participants discussed and adopted a list of recommendations which needed to be addressed during the meeting. This included the new recommendations as well as previous recommendations that needed to be reviewed and possibly revised.

The following protocol was used for the Technical Consultation: the meeting was structured to allow participants to discuss the proposed list of recommendations and these recommendations were revised, as needed, through group discussion. The final adoption of each recommendation was made by consensus - defined as the agreement by three quarters or more of the participants - provided that those who disagreed did not feel strongly about their position. Strong disagreements were recorded as such in the guideline. If the participants were unable to reach a consensus, the disputed recommendation, or any other decision, was put to a vote. A recommendation or decision stood if a simple majority (more than half of the participants) voted in support of it, unless the disagreement related to a safety concern, in which case the WHO Secretariat would choose not to issue a recommendation at all. WHO staff attending the meeting, external technical experts involved in the collection 
and grading of the evidence, and observers were not eligible to vote. In addition to discussing the scientific evidence and its quality, relevant applicability issues, costs and other judgements were also taken into consideration when formulating the final recommendations.

The strength of each recommendation was determined during the Technical Consultation. By default, the strength of the recommendations discussed was aligned initially with the quality of the evidence (i.e. at the start of the discussion, strong recommendations were based on evidence of 'moderate' and 'high' quality, while weak recommendations were based on evidence of 'low' and 'very low' quality). In addition to the quality of the evidence, the following factors were considered when determining the final recommendation and its strength: values and preferences, the magnitude of effect, the balance of benefits versus disadvantages, resource usage, and feasibility. Values and preferences, resource usage, and the feasibility of each recommendation were based on the experience and opinion of the GDG members. Balance worksheets were used to note and synthesize these considerations (Annex 3, Boxes 1 to 8 ) and record the reasons for changes made to the default strength of the recommendations.

\section{Document preparation and peer review}

Prior to the Technical Consultation, the guideline steering group prepared a preliminary version of this document using a guideline reporting template which had been developed as part of the WHO's GREAT project. The draft guideline was reviewed by Technical Consultation participants at the meeting in Montreux. During the meeting, the draft guideline was modified in line with participant deliberation and comments. Feedback received during the preliminary online consultation was also discussed and incorporated into the document where appropriate. After the meeting, members of the guideline steering group worked to ensure that a revised version of the document accurately reflected the deliberations and decisions of the participants. The revised draft guideline document was sent to two external peer reviewers and their inputs were carefully evaluated by the guideline steering group and document revisions made accordingly. The guideline steering group refrained from making substantive changes after the meeting in Montreux to the guideline scoping (such as the further expansion of the guideline scoping) or to the recommendations. The revised version was returned electronically to those who had attended the Technical Consultation for their approval.

\section{Results}

This guideline includes 32 recommendations for the prevention and treatment of $\mathrm{PPH}$. Seven of these recommendations are new, while the others have been revised in light of new evidence. Most of the previous 2007 and 2009 recommendations remain unchanged in essence, despite updates to the evidence base. The wording of the previous recommendations has been revised to enhance the clarity of the guidance provided. The recommendations included in this guideline are based on a total of 22 Cochrane systematic reviews summarized in 70 GRADE tables. Boxes 1 to 8 present the most up-to-date WHO recommendations for the prevention and treatment of PPH. Where applicable, remarks related to specific recommendations are also shown in these boxes and new recommendations are marked with asterisks. Narrative summaries of evidence supporting the recommendations are presented 
in the electronic appendix together with the corresponding GRADE tables (see the "WHO recommendations for preventing and treating PPH: evidence base" at www.who.int/reproductivehealth/publications/maternal_perinatal_health/ 9789241548502/en). Box 9 presents statements related to topics for which, according to the assessments of the GDG, there was insufficient evidence to issue a recommendation. Balance worksheets summarizing the values, preferences and judgements made about the strength of the recommendations are presented in Annex 3, Boxes 1 to 8 .

The development of these recommendations involved 130 stakeholders who participated in the online preliminary survey (representing all WHO regions), and 25 experts who participated in the WHO Technical Consultation.

\section{Recommendations for PPH prevention}

The contribution of each component of the 'active management of the third stage of labour' was examined in light of new available evidence, and relevant recommendations were made. Box 1 presents recommendations concerning the use of uterotonics for the prevention of PPH. All women giving birth should be offered uterotonics during the third stage of labour to prevent PPH and IM/IV oxytocin (10 IU) is recommended as the uterotonic drug of choice. Other injectable uterotonics (i.e. ergometrine/methylergometrine, or the fixed drug combination of oxytocin and ergometrine) and misoprostol are recommended as alternatives for the prevention of PPH in settings where oxytocin is unavailable. Box 2 contains recommendations related to cord management and uterine massage. The importance of controlled cord traction (CCT) was revisited because of new evidence. This intervention is now regarded as optional in settings where skilled birth attendants are available, and is contraindicated in settings where skilled attendants do not assist with births. Early cord clamping is generally contraindicated. Continuous uterine massage is not recommended as an intervention to prevent PPH for women who have received prophylactic oxytocin, because the massage may cause maternal discomfort, require a dedicated health professional, and may not lead to a reduction of blood loss. However, surveillance of the uterine tonus through abdominal palpation is recommended for all women for the early identification of postpartum uterine atony. Table 1 summarizes the recommendation status of the individual components of the active management of the third stage of labour. In summary, the GDG considered the use of uterotonics as the main intervention within the active management of third stage of labour package. In this context, the use of misoprostol for the prevention of PPH by community health care workers and lay health workers is supported in settings where skilled birth attendants are not present.

Recommendations for reducing blood loss during the third stage of labour in caesarean sections are presented in Box 3. Oxytocin is the recommended uterotonic drug for the prevention of PPH in caesarean sections. Cord traction is recommended in preference to manual removal when assisting placental delivery in caesarean sections.

\section{Recommendations for PPH treatment}

The use of uterotonics (oxytocin alone as the first choice) plays a central role in the treatment of PPH (see Boxes 4 and 5). Uterine massage is recommended for the treatment of PPH as soon as it is diagnosed (see Box 6) and the initial fluid resus- 
citation with isotonic crystalloids is recommended. The use of tranexamic acid is advised in cases of refractory atonic bleeding or persistent trauma-related bleeding (see Box 5). The use of intrauterine balloon tamponade is recommended for refractory bleeding or if uterotonics are unavailable. Bimanual uterine compression, external aortic compression, and the use of non-pneumatic anti-shock garments are recommended as temporizing measures until substantive care is available. If there is persistent bleeding and the relevant resources are available, uterine artery embolization should be considered. If bleeding persists despite treatment with uterotonic drugs and other conservative interventions, surgical intervention should be used without further delay.

If the third stage of labour lasts more than 30 minutes, CCT and IV/IM oxytocin (10 IU) should be used to manage the retained placenta. If the placenta is retained and bleeding occurs, the manual removal of the placenta should be expedited. Whenever the manual removal of the placenta is undertaken, a single dose of prophylactic antibiotics is recommended (see Box 7).

The GDG also issued recommendations related to the organization of PPH care (see Box 8 ). Health facilities delivering maternity services should adopt formal protocols for the prevention and treatment of PPH and for patient referral. The use of PPH treatment simulations for pre-service and in-service training programmes was recommended. Finally, the GDG recommended that the use of uterotonics for the prevention of PPH should be monitored and a specific indicator was suggested.

The GDG found insufficient evidence to recommend one route over another for the prevention of PPH with oxytocin, the use of recombinant factor VIla for the treatment of PPH, intraumbilical vein injection of oxytocin for treatment of retained placenta, and the antenatal distribution of misoprostol. The GDG also found insufficient evidence to recommend self-administration for the prevention of PPH and the measurement of blood loss over clinical estimation (see Box 9). 
Box 1: Recommendations for the prevention of PPH - uterotonics

1. The use of uterotonics for the prevention of PPH during the third stage of labour is recommended for all births. (Strong recommendation, moderate-quality evidence)

2. Oxytocin $(10 \mathrm{IU}, \mathrm{IV} / \mathrm{IM})$ is the recommended uterotonic drug for the prevention of PPH. (Strong recommendation, moderate-quality evidence)

3. In settings where oxytocin is unavailable, the use of other injectable uterotonics (e.g. ergometrine/methylergometrine or the fixed drug combination of oxytocin and ergometrine) or oral misoprostol $(600 \mu \mathrm{g})$ is recommended. (Strong recommendation, moderate-quality evidence)

4. In settings where skilled birth attendants are not present and oxytocin is unavailable, the administration of misoprostol ( $600 \mu \mathrm{g}$ PO) by community health care workers and lay health workers is recommended for the prevention of PPH. (Strong recommendation, moderatequality evidence)

\section{Remarks}

- Available comparisons are limited, but a significant difference between the benefits of oxytocin and ergometrine is unlikely. These recommendations place a high value on avoiding the adverse effects of ergometrine and assume a similar benefit from using oxytocin and ergometrine for the prevention of $\mathrm{PPH}$.

- Caution should be exercised when opting for ergot derivatives for the prevention of PPH as these drugs have clear contraindications in women with hypertensive disorders. Thus, it is probably safer to avoid the use of ergot derivatives in unscreened populations.

- Misoprostol ( $600 \mu \mathrm{g}$ PO) was regarded by the GDG as an effective drug for the prevention of PPH. However, the GDG considered the relative benefits of oxytocin compared to misoprostol in preventing blood loss, as well as the increased adverse effects of misoprostol compared to oxytocin. The GDG acknowledged that there is no evidence to show that a $600 \mu \mathrm{g}$ dose of misoprostol provides greater efficacy over a $400 \mu \mathrm{g} \mu \mathrm{g}$ dose. Lower doses have a lower side-effect profile but the efficacy of lower doses of misoprostol has not been evaluated sufficiently.

- The recommendations concerning alternative uterotonics should not detract from the objective of making oxytocin as widely accessible as possible.

- In view of past concerns regarding the community-level distribution of misoprostol and the potential for serious consequences of administration before birth, the GDG places emphasis on training persons administering misoprostol and monitoring community distribution interventions with scientifically sound methods and appropriate indicators. 
Box 2: Recommendations for the prevention of PPH - cord management and uterine massage

5. In settings where skilled birth attendants are available, CCT is recommended for vaginal births if the care provider and the parturient woman regard a small reduction in blood loss and a small reduction in the duration of the third stage of labour as important. (Weak recommendation, high-quality evidence)

6. In settings where skilled birth attendants are unavailable, CCT is not recommended. (Strong recommendation, moderate-quality evidence)

7. Late cord clamping (performed approximately 1 to 3 minutes after birth) is recommended for all births while initiating simultaneous essential newborn care. (Strong recommendation, moderate-quality evidence)

8. Early cord clamping ( $<1$ minute after birth) is not recommended unless the neonate is asphyxiated and needs to be moved immediately for resuscitation. (Strong recommendation, moderate-quality evidence)

9. Sustained uterine massage is not recommended as an intervention to prevent PPH in women who have received prophylactic oxytocin. (Weak recommendation, low-quality evidence)

10. Postpartum abdominal uterine tonus assessment for early identification of uterine atony is recommended for all women. (Strong recommendation, very-low-quality evidence)

\section{Remarks}

- Recommendations 5 and 6 are based on a large RCT in which oxytocin 10 IU was used for the prevention of PPH in all participants. Based on this evidence, CCT was regarded as safe when applied by skilled birth attendants as it provides small beneficial effects on blood loss (average reduction of $11 \mathrm{ml}$ on blood loss) and on the duration of the third stage of labour (average reduction of 6 minutes). The decision to implement CCT in the context of a prophylactic uterotonic drug should be discussed by the care provider and the woman herself.

- If ergot alkaloids are used for the prevention of PPH, then CCT to minimize placenta retention is regarded as essential.

- There is insufficient evidence to determine the benefit or risk of CCT when used in conjunction with misoprostol.

- $\quad$ CCT is the first intervention to treat retained placenta, therefore the teaching of CCT in medical and midwifery curricula is essential.

- The evidence base for recommendations for the timing of cord clamping includes both vaginal and caesarean births. The GDG considers this recommendation to be equally important for caesarean sections.

- Delayed clamping should be performed during the provision of essential newborn care. For essential newborn care and resuscitation, please refer to the WHO guidelines on neonatal resuscitation. (10)

- The recommendations for the timing of cord clamping apply equally to preterm and term births. The GDG considers the benefits of delayed clamping for preterm infants to be particularly important. 
- Some health professionals working in areas of high HIV prevalence have expressed concern regarding delayed cord clamping as part of management of the third stage of labour. These professionals are concerned that during placental separation, a partially detached placenta could be exposed to maternal blood and this could lead to a micro-transfusion of maternal blood to the baby. It has been demonstrated that the potential for maternal-to-child transmission of HIV can take place at three different points in time: micro-transfusions of maternal blood to the fetus during pregnancy (intra-uterine HIV transmission), exposure to maternal blood and vaginal secretions when the fetus passes through the birth canal in vaginal deliveries (intra-partum transmission), and during breastfeeding (postnatal infection). For this reason, the main intervention to reduce the maternal-to-child transmission is the reduction of maternal viral load through the use of antiretroviral drugs during pregnancy, childbirth and postnatal period. There is no evidence that delaying the cord clamping increases the possibility of HIV transmission from the mother to the newborn. Maternal blood percolates through the placental intervillous space throughout pregnancy with a relatively low risk of maternal fetal transmission before delivery. It is highly unlikely that separation of the placenta increases exposure to maternal blood, and is highly unlikely that it disrupts the fetal placental circulation (i.e. it is unlikely that during placenta separation the newborn circulation is exposed to maternal blood). Thus, the proven benefits of a 1 - 3 minute delay at least in clamping the cord outweigh the theoretical, and unproven, harms. Late cord clamping is recommended even among women living with HIV or women with unknown HIV status.

- There is a lack of evidence regarding the role of uterine massage for PPH prevention when no uterotonic drugs are used, or if a uterotonic drug other than oxytocin is used.

- Although the GDG acknowledged that one small study reported that sustained uterine massage and clot expulsion were associated with a reduction in the use of additional uterotonics, there is lack of robust evidence supporting other benefits. However, the GDG considered that routine and frequent uterine tone assessment remains a crucial part of immediate postpartum care, particularly for the optimization of early PPH diagnosis.

- Based on the most recent evidence, understanding of the contribution of each component of the active management of the third stage of labour package has evolved. The GDG considered that this package has a primary intervention: the use of an uterotonic. In the context of oxytocin use, CCT may add a small benefit, while uterine massage may add no benefit for the prevention of PPH. Early cord clamping is generally contraindicated. 
Table 1: Recommendation status of the individual components of the active management of the third stage of labour, based on who delivers the intervention

\begin{tabular}{|l|l|l|l|} 
& $\begin{array}{l}\text { Skilled } \\
\text { birth attendant }\end{array}$ & $\begin{array}{l}\text { Non-skilled } \\
\text { birth attendant }\end{array}$ & Self-administered \\
\hline Uterotonics & In favour & In favour & Research* \\
\hline Early cord clamping & Against & Against & Against \\
\hline Controlled cord traction & Conditional & Against & Against \\
\hline Continuous uterine massage & Against $^{* * *}$ & Against & Research \\
\hline
\end{tabular}

* Distribution of misoprostol during the antenatal period for self-administration during the third stage of labour

** Small reduction in blood loss and in the length of the third stage; adoption based on the values and preferences of the woman and the health care provider

*** Routine uterine tone assessment remains a vital part of clinical decision making and should be practised during the third stage of labour

**** Self-administered uterine massage in the absence of uterotonics

Box 3: Recommendations for the prevention of PPH in caesarean sections

11. Oxytocin (IV or IM) is the recommended uterotonic drug for the prevention of PPH in caesarean section. (Strong recommendation, moderate-quality evidence)

12. Cord traction is the recommended method for the removal of the placenta in caesarean section. (Strong recommendation, moderate-quality evidence)

\section{Remarks}

- The GDG noted that, in terms of blood loss, there was not enough evidence to recommend oxytocin infusion over IV bolus injection. However, due to concerns regarding adverse haemodynamic effects, the GDG considered that if an IV bolus injection is used, a slow injection rate is preferred and a rapid injection rate should be avoided.

- The GDG noted that the combination of an oxytocin infusion after an initial IV bolus of oxytocin after caesarean delivery reduces the need for additional uterotonic agents but does not affect the overall occurrence of major obstetric haemorrhage.

- The GDG noted that carbetocin is associated with a reduction in the use of additional uterotonic agents but with no difference in the occurrence of major obstetric haemorrhage. In addition, the GDG noted that the use of carbetocin is considerably more expensive than oxytocin. This remark is equally applicable to vaginal deliveries. 


\section{Box 4: Recommendations for the treatment of PPH - uterotonics}

13. Intravenous oxytocin is the recommended uterotonic drug for the treatment of PPH. (Strong recommendation, moderate quality evidence)

14. If intravenous oxytocin is unavailable, or if the bleeding does not respond to oxytocin, the use of intravenous ergometrine, oxytocin-ergometrine fixed dose, or a prostaglandin drug (including sublingual misoprostol, $800 \mu \mathrm{g}$ ) is recommended. (Strong recommendation, low-quality evidence)

\section{Remarks}

- The GDG recommended IV oxytocin as the first line uterotonic drug for the treatment of PPH, including when women have already received this drug for the prophylaxis of $\mathrm{PPH}$.

- The GDG recognized that IV oxytocin may not be available in all settings. It encourages health care decision-makers in these settings to strive to make oxytocin available.

- In settings where IV oxytocin is unavailable to women who have received prophylactic IM oxytocin during the third stage of labour, the GDG considered misoprostol to be a valid alternative.

- If PPH prophylaxis with misoprostol has been administered and if injectable uterotonics are unavailable, there is insufficient evidence to guide further misoprostol dosing and consideration must be given to the risk of potential toxicity.

- There is no added benefit to offering misoprostol simultaneously to women receiving oxytocin for the treatment of PPH (i.e. adjunct misoprostol).

- The GDG noted that the two largest trials of misoprostol for the treatment of PPH (Winikoff 2010, Blum 2010) reported the use of a $800 \mu \mathrm{g}$ dose administered sublingually. The majority of the GDG members agreed that $800 \mu \mathrm{g}$ is an acceptable sublingual misoprostol dose for the treatment of PPH, though some members of the GDG expressed concern related to the risk of hyperpyrexia associated with this dosage.

- If IV oxytocin has been used for the treatment of PPH and the bleeding does not stop, there is a paucity of data to recommend preferences for second line uterotonic drug treatment. Decisions in such situations must be guided by the experience of the provider, the availability of the drugs, and by known contraindications.

- In situations in which IM oxytocin can be administered and there is no possibility of IV treatment with ergot alkaloids/injectable prostaglandins, there is a paucity of data to recommend a preference of IM oxytocin over misoprostol or other uterotonics. Decisions in such situations must be guided by the experience of the provider, the availability of the drugs, and by known contraindications.

Box 5: Recommendations for the treatment of PPH - fluid resuscitation and tranexamic acid

15. The use of isotonic crystalloids is recommended in preference to the use of colloids for the intravenous fluid resuscitation of women with PPH. (Strong recommendation, low-quality evidence)

16. The use of tranexamic acid is recommended for the treatment of PPH if oxytocin and other uterotonics fail to stop the bleeding or if it is thought that the bleeding may be partly due to trauma. (Weak recommendation, moderate-quality evidence)

\section{Remarks}

- Evidence for the recommendation of tranexamic acid was extrapolated from the literature on surgery and trauma, which shows tranexamic acid to be a safe option for the treatment of trauma-related bleeding. 
Box 6: Recommendations for the treatment of PPH - manoeuvres and other procedures

17. Uterine massage is recommended for the treatment of PPH. (Strong recommendation, verylow-quality evidence)

18. If women do not respond to treatment using uterotonics, or if uterotonics are unavailable, the use of intrauterine balloon tamponade is recommended for the treatment of PPH due to uterine atony. (Weak recommendation, very-low-quality evidence)

19. If other measures have failed and if the necessary resources are available, the use of uterine artery embolization is recommended as a treatment for PPH due to uterine atony. (Weak recommendation, very-low-quality evidence)

20. If bleeding does not stop in spite of treatment using uterotonics and other available conservative interventions (e.g. uterine massage, balloon tamponade), the use of surgical interventions is recommended. (Strong recommendation, very-low-quality evidence)

21. The use of bimanual uterine compression is recommended as a temporizing measure until appropriate care is available for the treatment of PPH due to uterine atony after vaginal delivery. (Weak recommendation, very-low-quality evidence)

22. The use of external aortic compression for the treatment of PPH due to uterine atony after vaginal birth is recommended as a temporizing measure until appropriate care is available. (Weak recommendation, very-low-quality evidence)

23. The use of non-pneumatic anti-shock garments is recommended as a temporizing measure until appropriate care is available. (Weak recommendation, low-quality evidence)

24. The use of uterine packing is not recommended for the treatment of PPH due to uterine atony after vaginal birth. (Weak recommendation, very-low-quality evidence)

\section{Remarks}

- The GDG noted that the application of these interventions requires training and that maternal discomfort and complications associated with these procedures have been reported.

- Uterine massage as a therapeutic measure is defined as the rubbing of the uterus achieved through the manual massaging of the abdomen. This is typically sustained until the bleeding stops or the uterus contracts. The GDP considered that uterine massage should be started once PPH has been diagnosed.

- The initial rubbing of the uterus and expression of blood clots are not regarded as therapeutic uterine massage.

- When rating the recommendation \#17 as 'strong', the low cost and safety of uterine massage were taken into account.

- The use of balloon tamponade was considered by the GDG to be a measure that can potentially avoid surgery or as a temporizing measure while awaiting transfer to a higher level facility. The GDG acknowledges that balloon tamponade can be obtained with specific devices as well as with lower cost adaptations, including those based on the use of condoms and surgical gloves.

- The GDG noted that uterine artery embolization requires significant resources, in terms of the cost of the treatment, the facilities, and the training of health care workers.

- The GDG noted that conservative surgical approaches should be tried first. If these do not work, they should be followed by more invasive procedures. Compression sutures, for example, may be attempted as a first intervention, and if these fail, then uterine, utero-ovarian and hypogastric vessel ligation may be tried. If life-threatening bleeding continues even after ligation, then a subtotal (otherwise known as supracervical) or total hysterectomy should be performed.

- The GDG acknowledged that the level of health care provider skills will play a role in the selection and sequence of the surgical interventions. 
- External aortic compression has long been recommended as a potential life-saving technique, and mechanical compression of the aorta, if successful, slows blood loss. The GDG placed a high value on this procedure as a temporizing measure in the treatment of $\mathrm{PPH}$.

- The GDG noted that research evaluating the potential benefits and harms of non-pneumatic anti-shock garments is ongoing. Based on the evidence available, the GDG regarded non-pneumatic anti-shock garments as a temporizing measure while transfer is awaited.

- The GDG noted that there was no evidence of benefit of uterine packing and placed a high value on concerns regarding its potential harm.

Box 7: Recommendations for the treatment of retained placenta

25. If the placenta is not expelled spontaneously, the use of additional oxytocin (10 IU, IV/IM) in combination with controlled cord traction is recommended. (Weak recommendation, verylow-quality evidence)

26. The use of ergometrine for the management of a retained placenta is not recommended as this may cause tetanic uterine contractions which may delay the expulsion of the placenta. (Weak recommendation, very-low-quality evidence)

27. The use of prostaglandin E2 alpha (dinoprostone or sulprostone) in the management of retained placenta is not recommended. (Weak recommendation, very-low-quality evidence)

28. A single dose of antibiotics (ampicillin or first-generation cephalosporin) is recommended if manual removal of the placenta is practised. (Weak recommendation, very-low-quality evidence)

\section{Remarks}

- The GDG found no empirical evidence to support recommending the use of uterotonics for the management of a retained placenta in the absence of haemorrhage. The above recommendation was reached by consensus.

- The WHO guide, "Managing complications in pregnancy and childbirth" (WHO, 2007), states that if a placenta is not expelled within 30 minutes after the delivery of a baby, the woman should be diagnosed as having a retained placenta. Since there is no evidence for or against this definition, the delay used before this condition is diagnosed is left to the judgement of the clinician.

- The same WHO guide also suggests that in the absence of haemorrhage, the woman should be observed for a further 30 minutes after the initial 30 minutes, before the manual removal of the placenta is attempted. The GDG noted that spontaneous expulsion of the placenta can still occur, even in the absence of bleeding. A conservative approach is therefore advised and the timing of the manual removal of the placenta as a definitive treatment is left to the judgement of the clinician.

- The recommendation regarding the use of prostaglandin E2 is informed by a lack of evidence on this question and also by concerns related to adverse events, particularly cardiac events.

- Direct evidence of the value of antibiotic prophylaxis after the manual removal of the placenta was not available. The GDG considered indirect evidence of the benefit of prophylactic antibiotics from studies of caesarean section and abortion, as well as observational studies of other intrauterine manipulations.

- Current practice suggests that ampicillin or first-generation cephalosporins may be administered when the manual removal of the placenta is performed.

- This question was identified as a research priority for settings in which prophylactic antibiotics are not routinely administered and those with low infectious morbidity. 
Box 8: Health Systems and Organization of Care recommendations for the prevention and treatment of PPH

29. The use of formal protocols by health facilities for the prevention and treatment of PPH is recommended. (Weak recommendation, moderate-quality evidence)

30. The use of formal protocols for referral of women to a higher level of care is recommended for health facilities. (Weak recommendation, very-low-quality evidence)

31. The use of simulations of PPH treatment is recommended for pre-service and in-service training programmes. (Weak recommendation, very-low-quality evidence)

32. Monitoring the use of uterotonics after birth for the prevention of PPH is recommended as a process indicator for programmatic evaluation. (Weak recommendation, very-low-quality evidence)

\section{Remarks}

- Routine and frequent uterine tone assessment remains a crucial part of immediate postpartum care, particularly for optimizing the early diagnosis of PPH.

- The GDG acknowledged that the implementation of formal protocols is a complex process which will require the local adaptation of general guidelines.

- The GDG placed a high value on the costs of simulation programmes and acknowledged that there are different types of simulation programmes. Some programmes are hi-tech, computerized and costly while others are less expensive and more likely to be affordable in low- and middle-income countries. The GDG identified improvement in communication between health care providers and patients and their family members as an important priority in the training of health care providers in PPH management.

- The GDG recommended monitoring the use of prophylactic uterotonics. This recommendation is based on experience from other areas of health care, particularly child health, where content-based health indicators are common and regarded as useful for programmatic purposes. The suggested indicator is calculated as the number of women receiving prophylactic uterotonic drugs after birth divided by all women giving birth. 
Box 9: Statements related to topics for which there is insufficient evidence to issue a recommendation

A. There is insufficient evidence to recommend one oxytocin route over another for the prevention of PPH.

B. There is insufficient evidence to recommend the use of recombinant factor VIla for the treatment of $\mathrm{PPH}$.

C. There is insufficient evidence to recommend the use of intraumbilical vein injection of oxytocin as a treatment for retained placenta.

D. There is insufficient evidence to recommend the antenatal distribution of misoprostol to pregnant women for self-administration for the prevention of PPH.

E. There is insufficient evidence to recommend the measurement of blood loss over clinical estimation of blood loss.

\section{Remarks}

- The GDG noted that there are three ongoing trials in which the IV and IM routes for oxytocin administration are being compared for the prevention of PPH.

- The GDG considered there to be insufficient evidence to recommend the use of oxytocin infusion over IV bolus injection with regard to blood loss. However, in light of concerns about the potential adverse haemodynamic effects, the GDG considered that if IV bolus injection treatment is to be used then a slow injection rate is preferred and a rapid injection rate should be avoided.

- In the context of PPH, the GDP considered that the use of recombinant factor VIla should be limited to women with specific haematological indications. The group regarded the recombinant factor Vlla as a potentially life-saving drug, but noted that it is also associated with life-threatening side-effects. Moreover, recombinant factor VIla is expensive and may be difficult to administer.

- The GDG acknowledged that while there is a paucity of data to recommend intraumbilical vein injection of oxytocin as a treatment for retained placenta, the procedure itself has not been shown to cause harm and demonstrates a non-significant trend towards a lower risk of requiring the manual removal of the placenta.

- The GDG acknowledged that a number of countries have embarked on community-level programmes of misoprostol distribution and considered that this should be done in the context of research (where reliable data on coverage, safety and health outcomes can be collected).

- The GDG noted that all trials included in the systematic review on the measurement of blood loss were conducted in developed countries and views the applicability of this evidence to low- and middleincome countries as very uncertain. 


\section{Research implications}

The GDG identified important knowledge gaps that need to be addressed through primary research. In this guideline, recommendations based on evidence quality that was rated as 'very low' or 'low' require further research. Conversely, further research is not a priority for those recommendations based on evidence of 'moderate' or 'high' quality. Knowledge gaps identified in the 2007 and 2009 WHO documents were also reviewed. The identified knowledge gaps were prioritized by considering whether such research would be feasible, innovative, original, likely to promote equity, and contribute to the reduction of the burden of PPH. The main barriers to scaling up the intervention were also considered in this prioritization exercise.

The GDG noted that research is either planned or ongoing for some of the research priorities identified. However, there is no certainty that these investigations will provide conclusive results, and the topics have therefore remained listed as research priorities in this document.

\section{Key research priority}

In settings where the use of injectable uterotonics is not feasible, what are the effects of antenatal distribution of misoprostol to pregnant women for self-administration during the third stage of labour?

\section{Other research questions}

- What is the minimum effective dose of oxytocin for the prevention of PPH?

- What are the effects of IM oxytocin (versus IV oxytocin) for the prevention of $\mathrm{PPH}$ ?

- Can oxytocin be administered safely by unskilled attendants?

- What are the effects of buccal and sublingual use of oxytocin for the prevention of $\mathrm{PPH}$ ?

- What is the minimum effective dose of misoprostol for the prevention of PPH?

- What is the minimum effective dose of misoprostol for the treatment of $\mathrm{PPH}$ ?

- What are effects and safety of misoprostol as treatment for PPH, in women who received misoprostol as PPH prophylaxis?

- Should misoprostol be used in addition to oxytocin for PPH prevention?

- What are the effects of tranexamic acid in PPH treatment?

- What are the effects of uterine massage for the prevention of PPH?

- What are the effects of uterine massage to prevent PPH, where oxytocin is not available?

- What are the effects of uterine balloon or tamponade in the treatment of PPH?

- What are the effects of uterine massage to prevent PPH, where only misoprostol is available? 
- What are the effects of prophylactic antibiotics after manual extraction of the placenta as part of the treatment of retained placenta?

- What are the effects of the use of misoprostol for the treatment of retained placenta?

- What are effects of ergometrine (in combination or not with oxytocin) after caesarean section for the prevention of PPH?

- What is the optimal time for cord clamping in the context of physiologic and active management of third stage of labour?

- What is the appropriate time to administer oxytocin for PPH prevention, relative to cord clamping and placental delivery? (i.e. before/after cord clamping, before/after placenta delivery)

- Which clinical consequences of blood loss are of greatest value for the diagnosis and treatment of PPH?

- What is the role of lay health workers in management of PPH?

\section{Dissemination and implementation of the guideline}

The ultimate goal of this guideline is to improve the quality of care and health outcomes related to PPH. Therefore the dissemination and implementation of this guideline are crucial steps that should be undertaken by the international community and local health care services. The WHO Department of Reproductive Health and Research has adopted a formal knowledge-to-action framework for the dissemination, adaptation and implementation of guidelines (8). In addition to this framework, a list of priority actions was established during the WHO Technical Consultation which will be used by the WHO and other partners to foster the dissemination and implementation of this guideline (EB Box 2).

\section{Guideline dissemination and evaluation}

The recommendations in this guideline will be disseminated through a broad network of international partners, including WHO country and regional offices, ministries of health, WHO collaborating centres, other United Nations agencies, and non-governmental organizations. They will also be published on the WHO website and in The WHO Reproductive Health Library (11), where they will be accompanied by an independent critical appraisal based on the AGREE instrument (Appraisal of Guidelines Research and Evaluation) which can be found at http://www.agreecollaboration.org/instrument. A policy brief will also be developed for a wide range of policy-makers, programme managers and clinicians, and then disseminated through WHO country offices.

\section{Guideline implementation}

The successful introduction of evidence-based policies related to the prevention and management of PPH into national programmes and health care services depends on well-planned and participatory consensus-driven processes of adaptation and imple- 
mentation. These processes may include the development or revision of existing national guidelines or protocols based on this document.

The recommendations contained in the present guideline should be adapted into locally-appropriate documents that are able to meet the specific needs of each country and health service. Modifications to the recommendations, where necessary, should be limited to weak recommendations and justifications for any changes made in an explicit and transparent manner.

An enabling environment should be created for the use of these recommendations (for example, by widening the availability of uterotonics), including changes in the behaviour of health care practitioners to enable the use of evidence-based practices. Local professional societies may play important roles in this process and an allinclusive and participatory process should be encouraged. The WHO's Department of Reproductive Health and Research has published specific guidance on the introduction of the WHO's reproductive health guidelines and tools in national programmes.

\section{Applicability issues}

\section{Anticipated impact on the organization of care and resources}

The evidence-based prevention and management of PPH can be achieved with the use of relatively inexpensive drugs. However, the GDG noted that the following issues should be considered before the recommendations made in this current guideline are applied:

- Women should not be left alone during the first hours after delivery of the baby and the placenta

- In settings where oxytocin is used, attention should be paid to the oxytocin cold chain (i.e. the requirements of a temperature-controlled supply chain)

- Health services adopting late cord clamping should also adopt strategies to identify (and if necessary to treat) neonatal jaundice

\section{Monitoring and evaluating the guideline implementation}

The implementation of the recommendations in this guideline should be monitored at the health-service level. Interrupted time series, clinical audits or criterion-based clinical audits could be used to obtain relevant data related to the management of PPH. Clearly-defined review criteria and indicators are needed and these could be associated with locally-agreed targets. The GDG strongly recommends that the coverage of prophylactic uterotonics be used as a process indicator for the monitoring and prevention of PPH.

- Prophylactic Uterotonic Coverage Indicator: The suggested indicator is calculated as the number of women receiving prophylactic uterotonics during the third stage of labour divided by all women giving birth

This indicator provides an overall assessment of adherence to a key recommendation included in this guideline. The use of other locally-agreed and more specific indica- 
tors (e.g. the assessment of the use of specific uterotonics) may be necessary to obtain a more complete assessment of the quality of care related to the prevention and treatment of PPH. WHO has developed specific guidance for evaluating the quality of care for severe maternal complications (including PPH) based on the near-miss and criterion-based clinical audit concepts (13).

\section{Updating the guideline}

This guideline will be updated in 2017 or following the identification of new evidence that indicates a need to revise these recommendations. WHO welcomes suggestions regarding additional questions for inclusion in the updated guideline. Please e-mail your suggestions to: reproductivehealth@who.int.

\section{References}

1. Khan KS, Wojdyla D, Say L, Gülmezoglu AM, Van Look PF. WHO analysis of causes of maternal death: A systematic review. Lancet. 2006;367 (9516): 1066-74.

2. Campbell OM, Graham WJ. Lancet Maternal Survival Series Steering Group. Strategies for reducing maternal mortality: getting on with what works. Lancet. 2006;368 (9543): 1284-99.

3. World Health Organization. World Health Organization multicountry survey on maternal and newborn health. Geneva: WHO; 2012

4. World Health Organization. Managing complication in pregnancy and childbirth: a guide for midwives and doctors. Geneva: WHO; 2000. Available from: http://www.who.int/reproductivehealth/publications/maternal_perinatal_ health/9241545879/en/index.html

5. Begley CM, Gyte GM, Devane D, McGuire W, Weeks A. Active versus expectant management for women in the third stage of labour. Cochrane Database Syst Rev. 2011(11). Available from: http: / / onlinelibrary.wiley.com/ doi/10.1002/14651858.CD007412.pub3/abstract

6. World Health Organization. WHO recommendations for the prevention of postpartum haemorrhage. Geneva: WHO; 2007. Available from: http://whqlibdoc. who.int/hq/2007/WHO_MPS_07.06_eng.pdf

7. World Health Organization. WHO guidelines for the management of postpartum haemorrhage and retained placenta. Geneva: WHO; 2009. Available from: http://whqlibdoc.who.int/publications/2009/9789241598514_eng.pdf

8. World Health Organization. Knowledge to action framework and the G.R.E.A.T project. Geneva: WHO; 2010. Available from: http://www.who.int/reproductivehealth/topics/best_practices/greatproject_KTAframework/en/index.html

9. World Health Organization. WHO Handbook for guideline development. Geneva: WHO; 2012. Available from: http://apps.who.int/iris/bitstre am/10665/75146/1/9789241548441_eng.pdf 
10. World Health Organization. Guidelines on basic newborn resuscitation. Geneva: WHO; 2012. Available from: http://apps.who.int/iris/bitstre am/10665/75157/1/9789241503693_eng.pdf

11. World Health Organization. The WHO Reproductive Health Library. Geneva: WHO. Available from: www.who.int/rhl

12. World Health Organization. Introducing WHO's sexual and reproductive health guidelines and tools into national programmes: principles and process of adaptation and implementation. Geneva: WHO; 2007. Available from: http://www. who.int/reproductivehealth/publications/general/RHR_07_09/en/index.html

13. World Health Organization. Evaluating the quality of care for severe pregnancy complications: the WHO near-miss approach for maternal health. Geneva: WHO; 2011. Available from: http: / /whqlibdoc.who.int/publications/2011/9789241502221_eng.pdf

The full list of references that support the recommendations is included in the document entitled "WHO recommendations for postpartum haemorrhage: evidence base" and can be accessed online at: www.who.int/reproductivehealth/publications/maternal_perinatal_health/9789241548502/en/ 


\section{Annex 1. External experts, WHO staff involved in the preparation of the guideline, and summary of decla- rations of interest}

A. Guideline Development Group (participants in the WHO Technical Consultation)

\section{Members (WHO External Advisers)}

Professor Hany Abdel-Aleem

Professor of Obstetrics and Gynecology

Women's Health Center

Assiut University Hospital

Assiut

Egypt

\section{Dr Catherine Deneux-Tharaux}

Medical Epidemiologist and Researcher Inserm U953

Recherche épidémiologique en santé périnatale et santé des femmes et des enfants

Hôpital Tenon

Paris

France

\section{Dr Bukola Fawole}

Senior Lecturer

Department of Obstetrics and Gynaecology

College of Medicine

University of Ibadan

Ibadan

Nigeria

\section{Dr Atf Ghérissi}

Maître Assistante Universitaire en Sciences de

l'Education appliquées à la Santé

Ecole Supérieure des Sciences et Techniques de la Santé Université

Tunis-El Manar

Tunisia

\section{Ms Gill Gyte}

Research Associate

Cochrane Pregnancy and Childbirth Group

University of Liverpool

Liverpool Women's Hospital NHS Trust

Crown Street

United Kingdom

\author{
Dr Justus Hofmeyr \\ Director \\ Effective Care Research Unit \\ University of the Witwatersrand/ \\ University of Fort Hare/ \\ Eastern Cape Department of Health \\ Amalinda Drive \\ Private Bag X9047 \\ East London \\ Eastern Cape 5201 \\ South Africa \\ Dr Simon Lewin \\ Senior Researcher \\ Global Health Unit \\ Norwegian Knowledge Centre for the Health \\ Services \& Medical Research Council, South Africa \\ Oslo \\ Norway \\ Professor Syeda Batool Mazhar \\ Professor of Obstetrics and Gynaecology \\ Mother and Child Health Centre (MCH) Pakistan \\ Institute of Medical Sciences \\ Islamabad \\ Pakistan

\section{Professor Suneeta Mittal} \\ Professor of Obstetrics and Gynecology \\ Officer-in-Charge of the WHO Collaborating \\ Centre for Research on Human Reproduction \\ All India Institute of Medical Sciences \\ New Delhi \\ India \\ Dr Enrique Oyarzun \\ Chairman \\ Department Obstetrics and Gynaecology \\ Facultad de Medicina \\ Pontificia Universidad Católica de Chile \\ Santiago \\ Chile
}


Dr Zahida Qureshi

Senior Lecturer

Department of Obstetrics and Gynaecology

University of Nairobi

Nairobi

Kenya

Professor Hamid Rushwan

Chief Executive

International Federation of Gynecology and Obstetrics

FIGO House, Suite 3

Waterloo Court, 10 Theed Street

London

SE1 8ST

United Kingdom

Dr Jeffrey Michael Smith

Director, Maternal Health, MCHIP

Washington

USA

\section{Dr Tran Son Thach}

Perinatal Epidemiologist

Australian Research Centre for Health of Women and Babies

Discipline of Obstetrics and Gynaecology

The University of Adelaide

Women's and Children's Hospital

King William Road

Australia

\section{Dr Dilys Walker}

Associate Professor

Department of Global Health and Obstetrics \&

Gynecology

University of Washington

Ninth \& Jefferson Building, Harborview Medical Center Seattle, WA

USA

\section{Observers}

\section{Ms Deborah Armbruster}

Senior Maternal and Newborn Health Advisor Center for Population, Health and Nutrition United States Agency for International Development Washington D.C.

USA

\section{Ms Jennifer Blum}

Gynuity Health Projects

New York

USA

\section{Ms Claire Glenton}

Senior Researcher

Nordic Cochrane Centre, Norwegian Branch/

Global Health Unit

Norwegian Knowledge Centre for the Health Services Oslo

Norway

\section{Dr Sarah Rosenbaum}

Norwegian Knowledge Centre for the Health Services Oslo

Norway

\section{Ms Mary Ellen Stanton}

Senior Reproductive Health Advisor

Center for Population, Health and Nutrition

United States Agency for International Development Washington D.C.

USA

\section{Ms Clare Waite}

Project Manager

Misoprostol for Post-Partum Haemorrhage in Low

Resource Settings

International Federation of Gynecology and

Obstetrics

FIGO House

London

United Kingdom

Dr Beverly Winikoff

Gynuity Health Projects

New York

USA 


\section{WHO Regional and Country Offices}

\section{AFRO}

\section{Dr Alicia Carbonell}

National Professional Officer

Making Pregnancy Safer and Reproductive Health

Bureau de pays de l'OMS

PO Box CP 377

Maputo

Mozambique

\section{SEARO}

\section{Dr Narimah Awin}

Medical Officer

Making Pregnancy Safer and Reproductive Health Department of Family and Research

World Health Organization

Regional Office for South-East Asia

World Health House, Indraprastha Estate

Mahatma Gandhi Marg

New Delhi 110002

India

WPRO

Dr Hiromi Obara

Medical Officer

Maternal and Child Health and Nutrition

Building Healthy Communities and Populations

World Health Organization

Regional Office for the Western Pacific

P.O. Box 2932

1000 Manila

Philippines

\section{External Secretariat}

\section{Dr Edgardo Abalos}

Centro Rosarino de Estudios Perinatales (CREP)

Rosario

Argentina

\section{Dr Virginia Diaz}

Centro Rosarino de Estudios Perinatales (CREP)

Rosario

Argentina

Dr Natasha Hezelgrave

Academic Clinical Fellow, Obstetrics and Gynaecology Kings College London

Guy's \& St Thomas' NHS Foundation Trust

London

United Kingdom

\section{WHO Secretariat}

\section{Dr Michael Mbizvo}

Director

Department of Reproductive Health and Research

Dr Ana Pilar Betran

Medical Officer

Improving Maternal and Perinatal Health

Research, Evidence and Norms

Department of Reproductive Health and Research

Dr Metin Gülmezoglu

Lead Specialist

Improving Maternal and Perinatal Health

Research, Evidence and Norms

Department of Reproductive Health and Research

Dr Matthews Mathai

Coordinator

Epidemiology, Monitoring and Evaluation

Department of

Maternal, Newborn, Child and Adolescent

Health (MCA)

Dr João Paulo Souza

Medical Officer

Improving Maternal and Perinatal Health Research, Evidence and Norms

Department of Reproductive Health and Research

\section{Dr Joshua Vogel}

Improving Maternal and Perinatal Health Research, Evidence and Norms

Department of Reproductive Health and Research

\section{Dr Mariana Widmer}

Technical officer

Improving Maternal and Perinatal Health Research, Evidence and Norms

Department of Reproductive Health and Research

\section{B. Guideline Steering Group}

Dr A. Metin Gülmezoglu (WHO)

Dr Matthews Mathai (WHO)

Dr João Paulo Souza (WHO)

Dr Edgardo Abalos (CREP)

Dr Virginia Diaz (CREP)

Dr Natasha Hezelgrave (KCL) 


\section{Summary of the declarations of interest: Members of the GDG}

\begin{tabular}{|c|c|c|c|c|c|c|c|c|}
\hline \multirow[t]{2}{*}{ Name } & \multirow[t]{2}{*}{ Region } & \multirow[t]{2}{*}{ Country } & \multicolumn{4}{|c|}{$\begin{array}{l}\text { Declaration of Conflict } \\
\text { of interest } \\
\text { (please indicate 'yes' or } \\
\text { 'no' for each section) }\end{array}$} & \multirow[t]{2}{*}{$\begin{array}{l}\text { Advice from } \\
\text { Legal Department } \\
\text { (please indicate } \\
\text { 'yes' or 'no') }\end{array}$} & \multirow{2}{*}{$\begin{array}{l}\text { Meeting } \\
\text { restriction: } \\
\text { Please indicate } \\
\text { see below for } \\
\text { explanation) }\end{array}$} \\
\hline & & & A & B & C & D & & \\
\hline $\begin{array}{l}\text { Professor Hany } \\
\text { Abdel-Aleem }\end{array}$ & EMRO & Egypt & $Y$ & $\mathrm{~N}$ & $\mathrm{~N}$ & $\mathrm{~N}$ & $\mathrm{~N}$ & N \\
\hline $\begin{array}{l}\text { Dr Catherine } \\
\text { Deneux-Tharaux }\end{array}$ & EURO & France & $Y$ & $\mathrm{~N}$ & $\mathrm{~N}$ & $\mathrm{~N}$ & $\mathrm{~N}$ & $\mathrm{~N}$ \\
\hline Dr Bukola Fawole & AFRO & Nigeria & $N$ & $\mathrm{~N}$ & $\mathrm{~N}$ & $\mathrm{~N}$ & $\mathrm{~N}$ & N \\
\hline Dr Atf Ghérissi & EMRO & Tunisia & $N$ & $\mathrm{~N}$ & $\mathrm{~N}$ & $\mathrm{~N}$ & $\mathrm{~N}$ & N \\
\hline Ms Gill Gyte & EURO & UK & $Y$ & $\mathrm{~N}$ & $\mathrm{~N}$ & $\mathrm{~N}$ & $\mathrm{~N}$ & $\mathrm{~N}$ \\
\hline Dr Justus Hofmeyr & AFRO & $\begin{array}{l}\text { South } \\
\text { Africa }\end{array}$ & $Y$ & $\mathrm{~N}$ & $\mathrm{~N}$ & $N$ & $N$ & $\mathrm{~N}$ \\
\hline Dr Simon Lewin & EURO & $\begin{array}{l}\text { Norway/ } \\
\text { South } \\
\text { Africa }\end{array}$ & $N$ & $\mathrm{~N}$ & $\mathrm{~N}$ & $\mathrm{~N}$ & $\mathrm{~N}$ & N \\
\hline $\begin{array}{l}\text { Professor Syeda } \\
\text { Batool Mazhar }\end{array}$ & EMRO & Pakistan & $\mathrm{N}$ & $\mathrm{N}$ & $\mathrm{N}$ & $\mathrm{N}$ & $\mathrm{N}$ & N \\
\hline $\begin{array}{l}\text { Dr Enrique } \\
\text { Oyarzun }\end{array}$ & AMRO & Chile & $\mathrm{N}$ & $\mathrm{N}$ & $N$ & $N$ & $\mathrm{~N}$ & N \\
\hline Dr Zahida Qureshi & AFRO & Kenya & $\mathrm{Y}$ & $\mathrm{N}$ & $N$ & $\mathrm{~N}$ & $\mathrm{~N}$ & N \\
\hline $\begin{array}{l}\text { Professor } \\
\text { Hamid Rushwan }\end{array}$ & EURO & Sudan/UK & $\mathrm{N}$ & $\mathrm{N}$ & $\mathrm{N}$ & $\mathrm{N}$ & $\mathrm{N}$ & N \\
\hline $\begin{array}{l}\text { Dr Jeffrey Michael } \\
\text { Smith }\end{array}$ & AMRO & USA & $\mathrm{N}$ & $\mathrm{N}$ & $\mathrm{N}$ & $\mathrm{N}$ & $\mathrm{N}$ & $\mathrm{N}$ \\
\hline Dr Tran Son Thach & WPRO & $\begin{array}{l}\text { Vietnam / } \\
\text { Australia }\end{array}$ & $Y$ & $\mathrm{~N}$ & $N$ & $N$ & $\mathrm{~N}$ & N \\
\hline Dr Dilys Walker & AMRO & USA & $Y$ & $\mathrm{~N}$ & $N$ & $\mathrm{~N}$ & $\mathrm{~N}$ & N \\
\hline
\end{tabular}

A: Involved in academic work related to the topic of the meeting/guideline

B: Declared any commercial financial interest, related to the topic of the meeting/guideline

C: Declared any commercial financial interest, not directly related to the topic of the meeting/guideline

D: Declared non-commercial interest or grants related to the topic of the meeting/guideline 


\section{Annex 2. Critical outcomes for decision making}

\section{PPH prevention}

\section{Critical outcomes}

Fewer maternal deaths

Fewer events of severe PPH (blood loss $>1000 \mathrm{ml}$ )

Less use of blood transfusion

Important outcomes

Fewer admissions to intensive care unit

Blood loss $\geq 500 \mathrm{ml}$

Additional uterotonics

Mean blood loss

Postpartum anaemia

Breastfeeding

Less anaemia in infancy

Any side effect of intervention

Any side effect requiring treatment

Nausea

Vomiting

Diarrhoea

Headache

Abdominal pain

High blood pressure

Shivering

Maternal temperature $\geq 38^{\circ} \mathrm{C}$

Maternal temperature $\geq 40{ }^{\circ} \mathrm{C}$

\section{PPH treatment}

\section{Critical outcomes}

Additional blood loss $\geq 500 \mathrm{ml}$

Additional blood loss $\geq 1000 \mathrm{ml}$

Blood transfusion

Additional uterotonics

Invasive non-surgical interventions

Surgical interventions (including hysterectomy)

Maternal temperature $\geq 40{ }^{\circ} \mathrm{C}$

Procedure-related complications

Infections

Severe morbidity

Maternal transfer

Reduction of time from decision-making to implementation

Availability of drugs and treatment

Important outcomes

Accuracy in blood loss assessment

Mean blood loss

Postpartum anaemia

Additional non-surgical interventions (e.g. external aortic compression and compression garments)

Artery embolization

Nausea, vomiting or shivering

Maternal temperature $\geq 38{ }^{\circ} \mathrm{C}$

Delayed initiation of breastfeeding

Prolonged hospitalization 


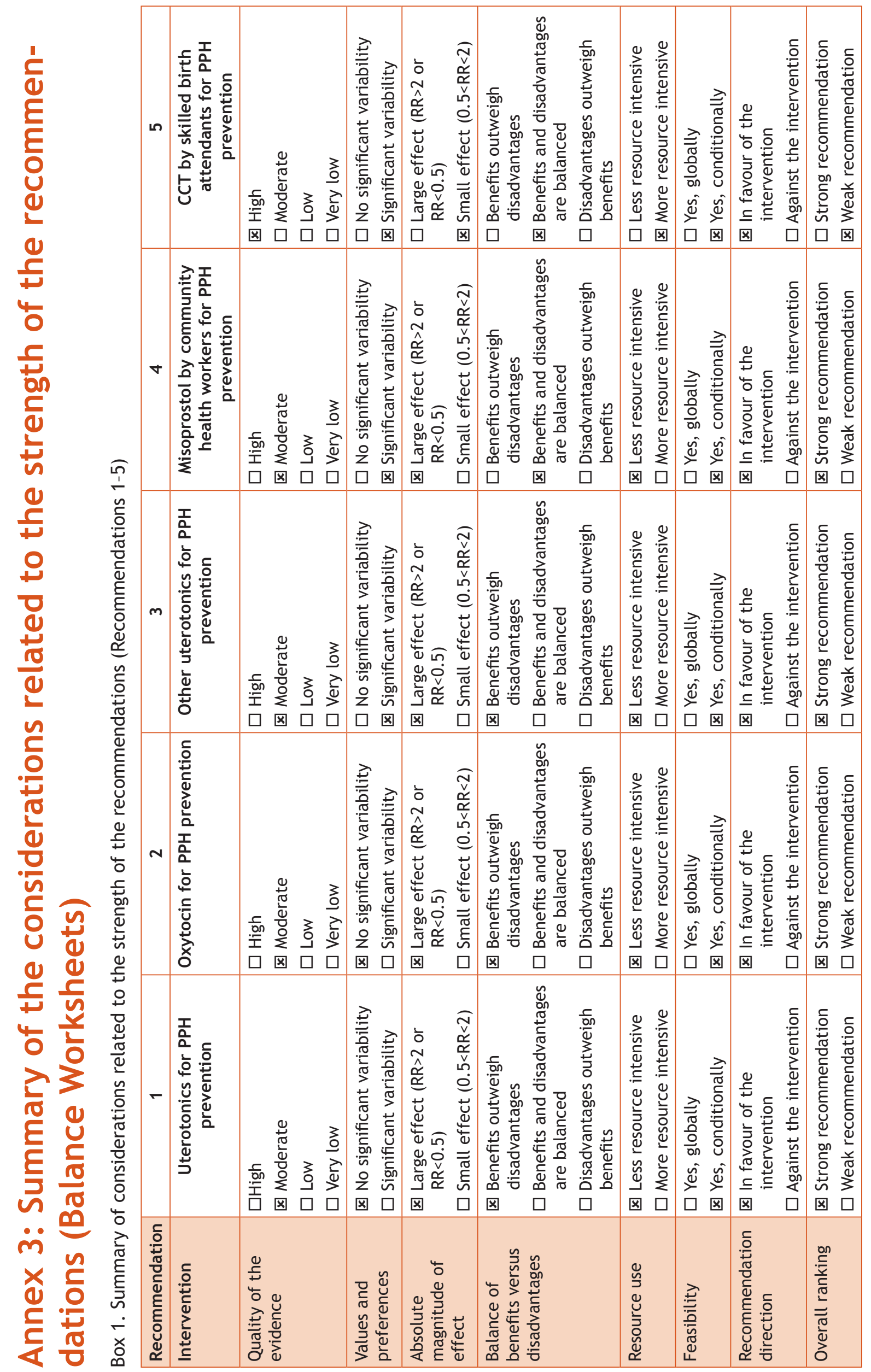




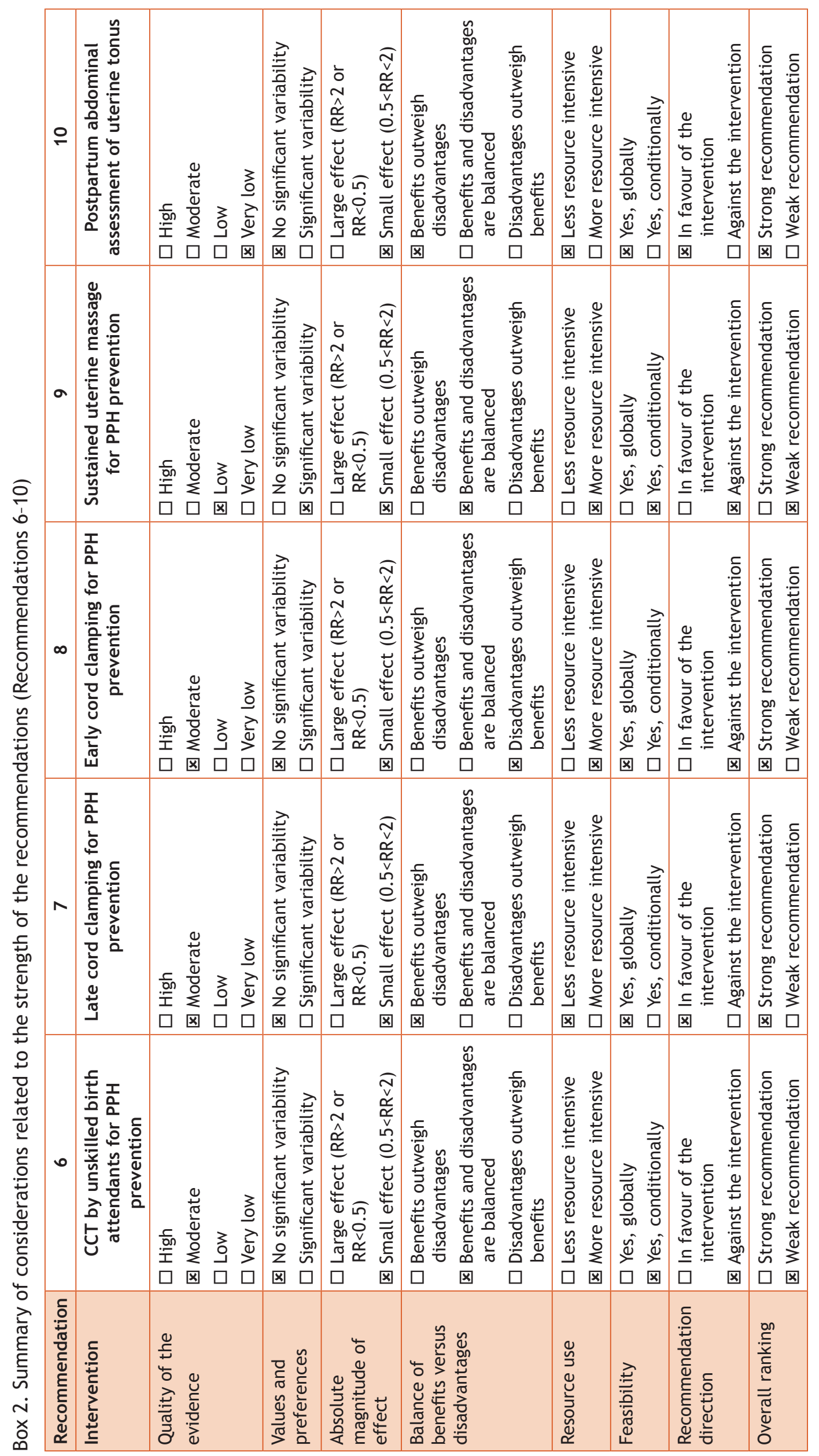




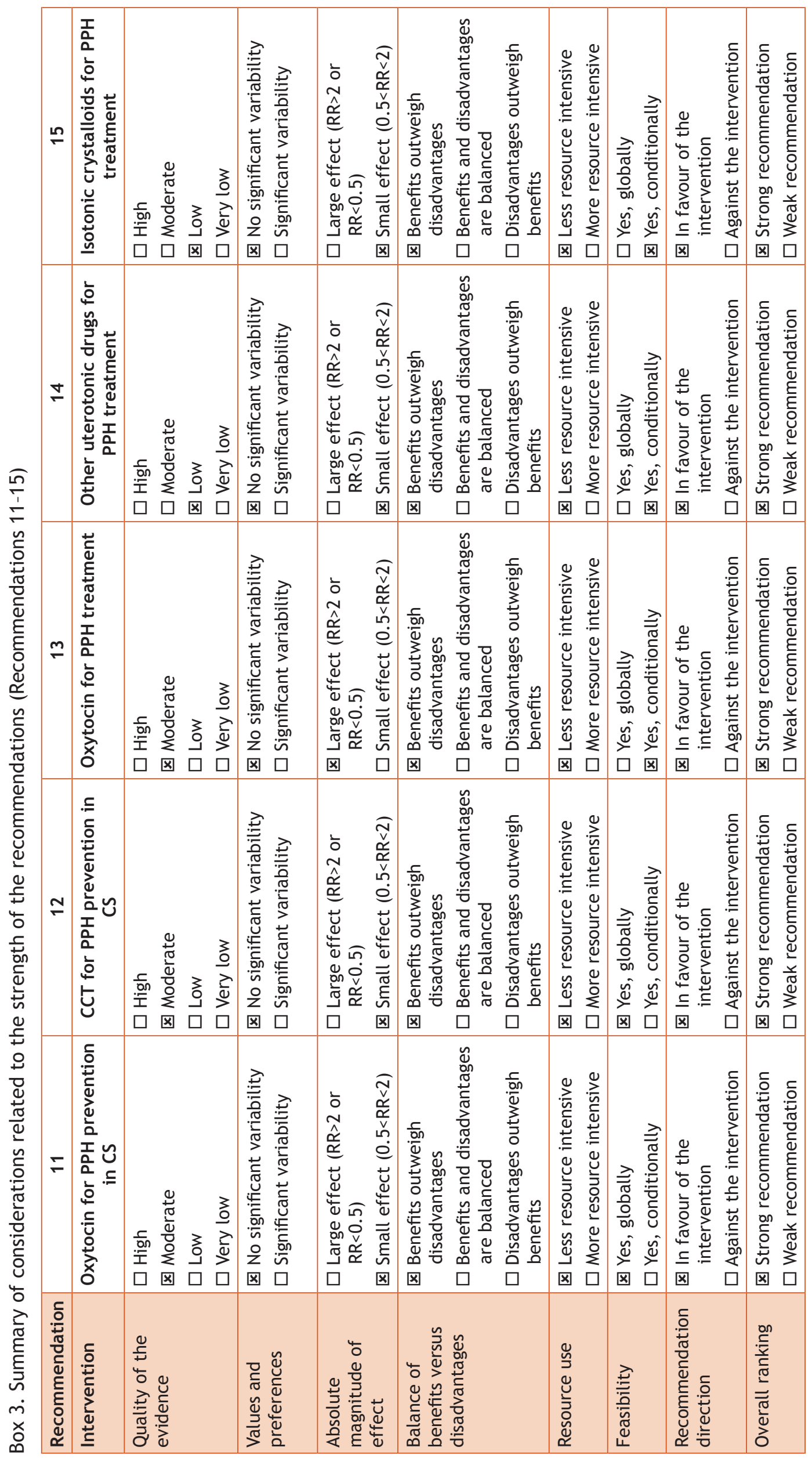




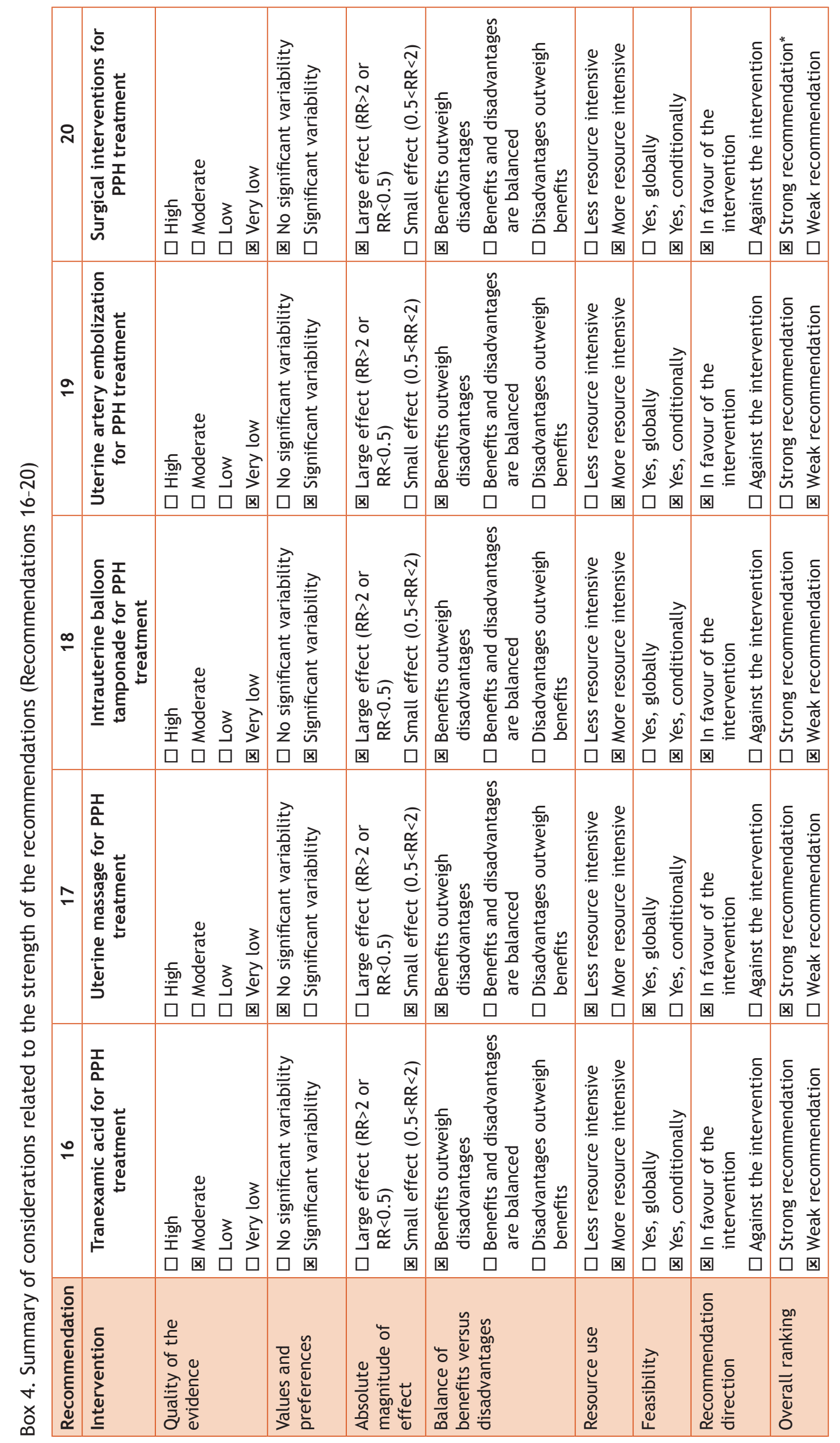




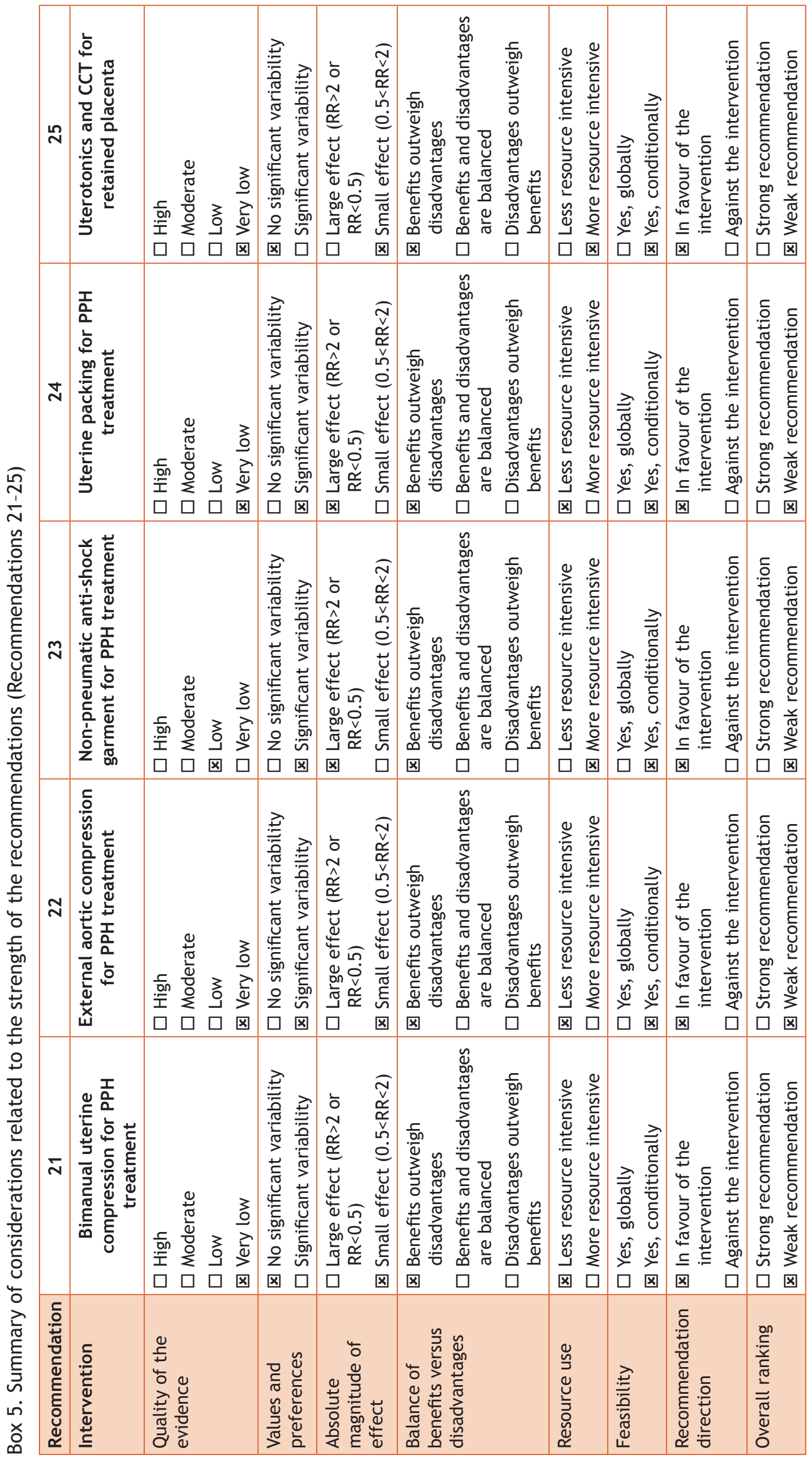




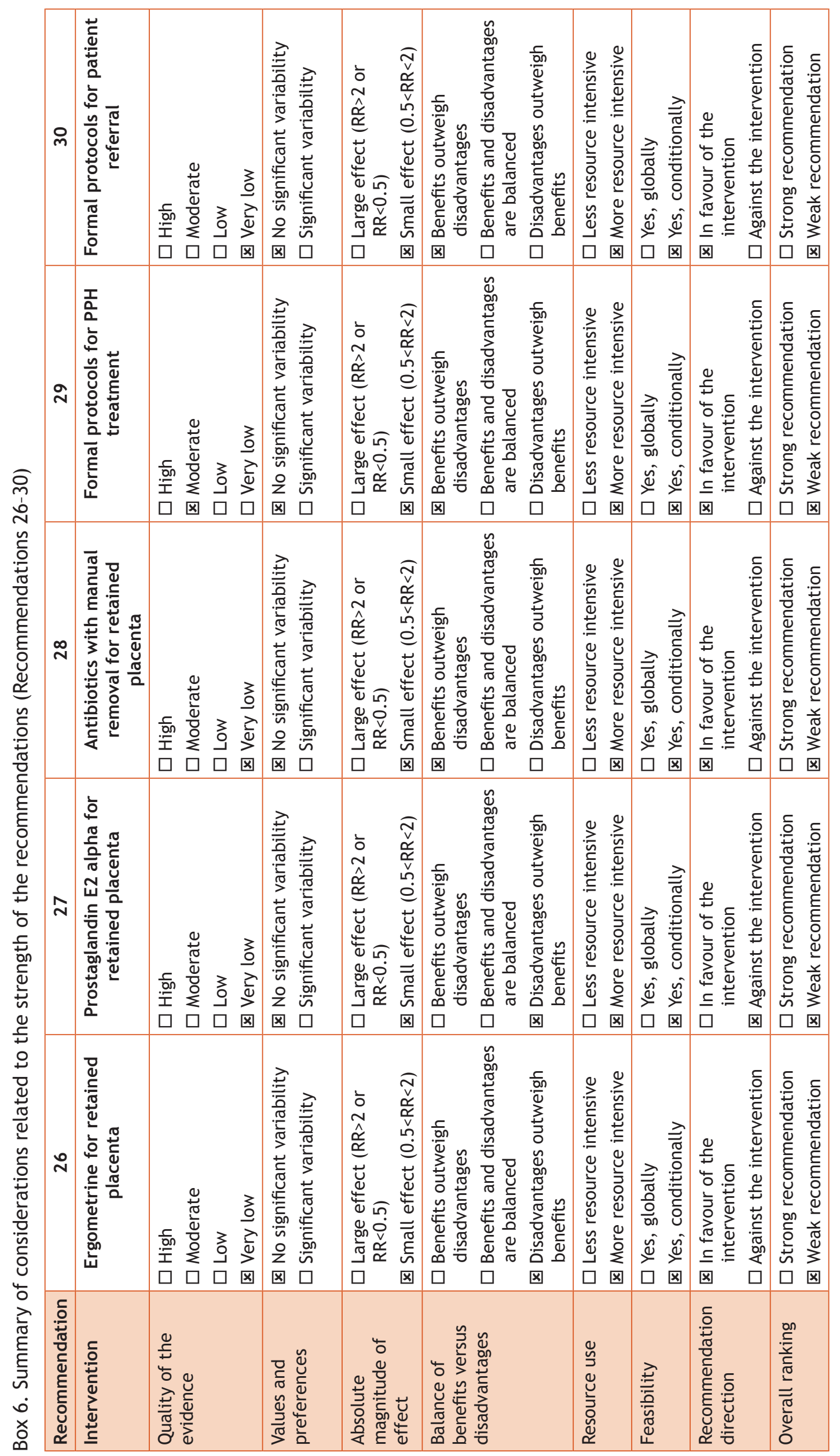




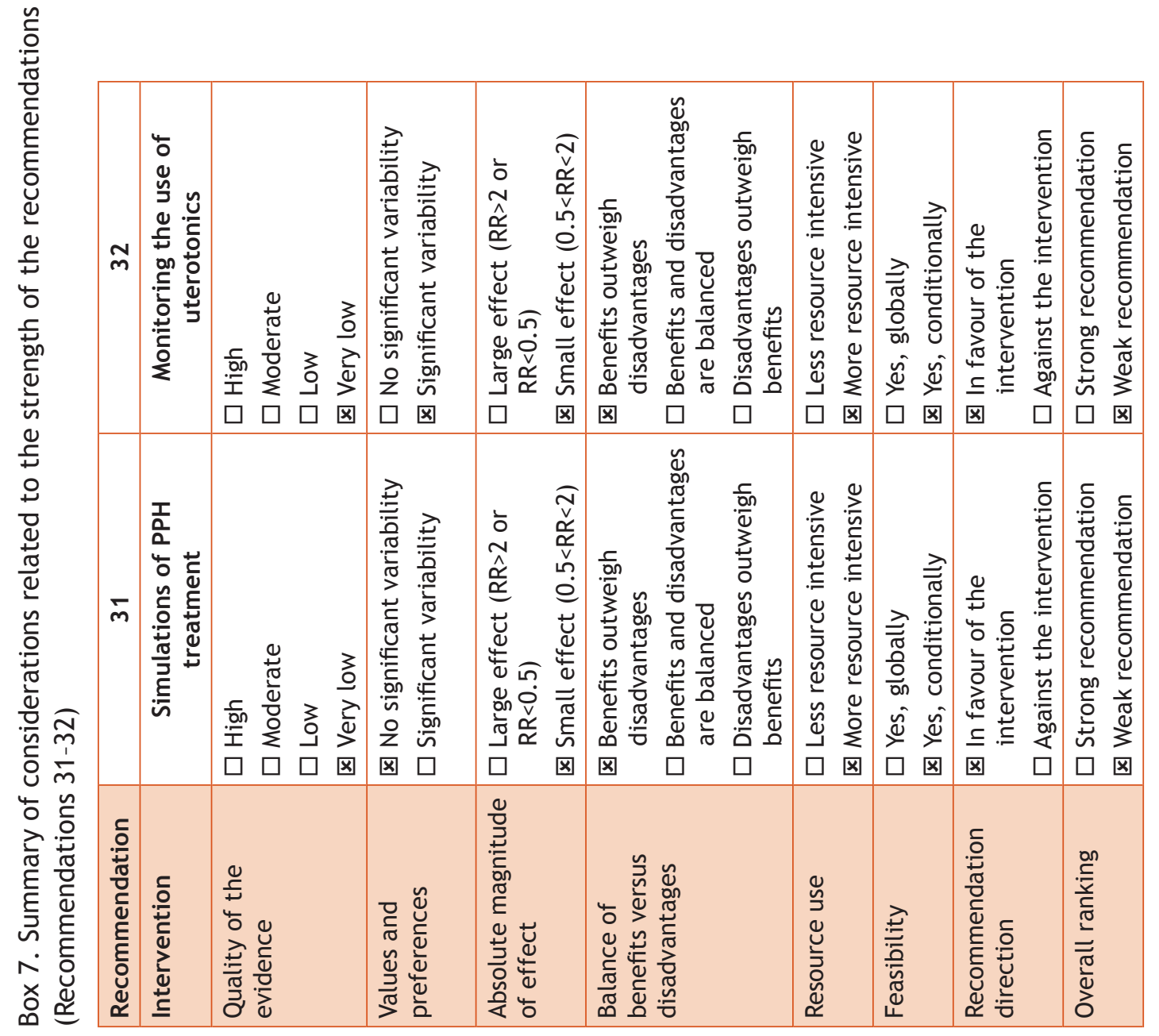




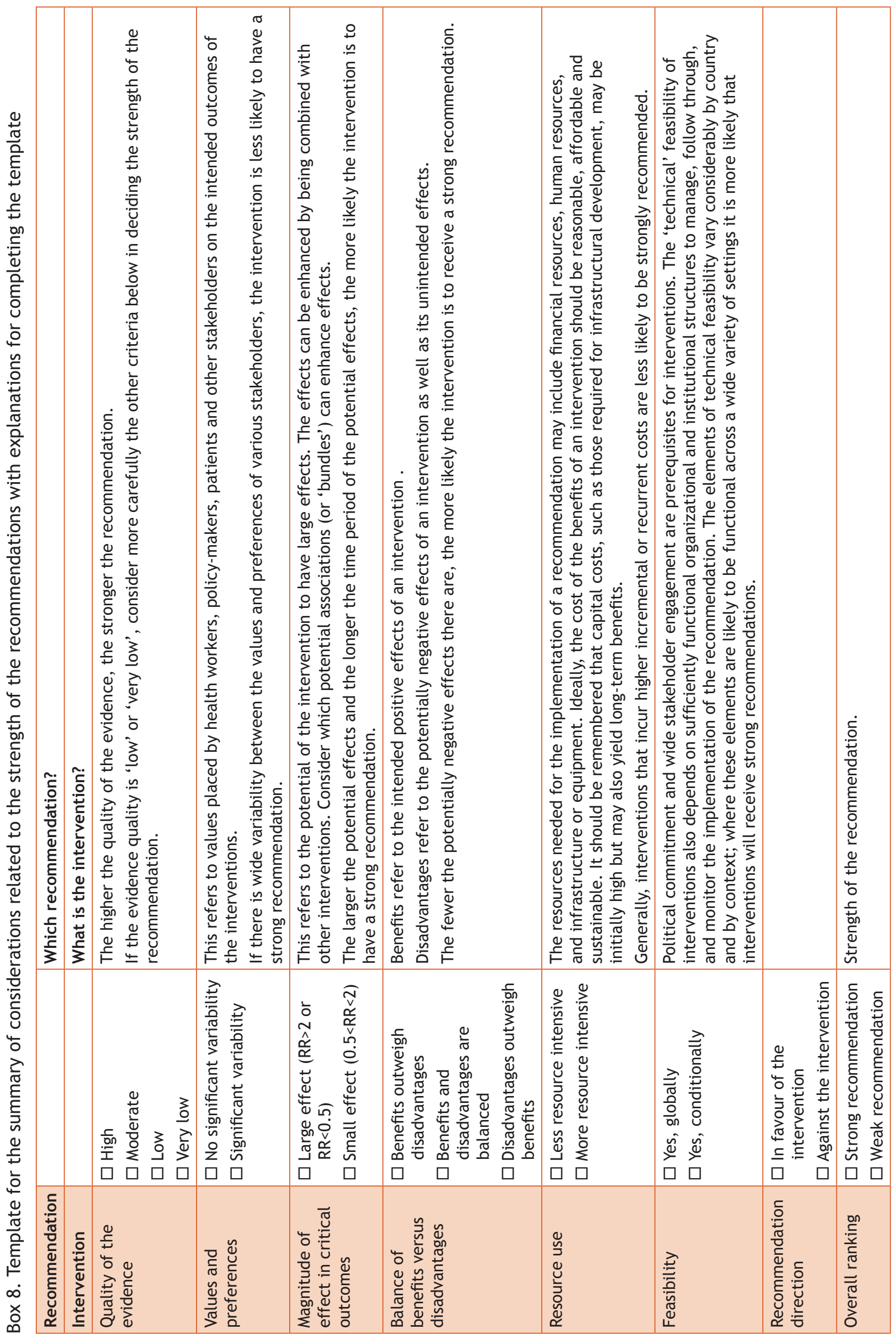


